\title{
Dynamic Evaluation of Mesh Resolution and Its Application in Hybrid LES/RANS Methods
}

\section{Journal Article}

\section{Author(s):}

Xiao, Heng; Wang, Jianxun; Jenny, Patrick

Publication date:

2014-07

\section{Permanent link:}

https://doi.org/10.3929/ethz-b-000087239

\section{Rights / license:}

In Copyright - Non-Commercial Use Permitted

\section{Originally published in:}

Flow, Turbulence and Combustion 93(1), https://doi.org/10.1007/s10494-014-9541-9 


\title{
Dynamic Evaluation of Mesh Resolution and Its Application in Hybrid LES/RANS Methods
}

\author{
Heng Xiao · Jianxun Wang • Patrick Jenny
}

Received: 30 April 2013 / Accepted: 28 April 2014 / Published online: 22 May 2014

(C) Springer Science+Business Media Dordrecht 2014

\begin{abstract}
In this work, we investigate a resolution evaluation criterion based on the ratio between turbulent length-scales and grid spacing within the context of dynamic resolution evaluation in hybrid LES/RANS simulations. A modified version of the commonly used length-scale criterion is adopted. The modified length-scale criterion is evaluated for a plane channel flow and compared to the criterion based on two-point correlations. Simulation results show qualitative agreement between the two criteria and physical predictions from both resolution indicators. These observations are confirmed by simulations of flows over periodic hills. It is further demonstrated that the length-scale based criterion is relatively less sensitive on variation of model parameters compared to criteria based on resolved percentage of turbulent quantities. The improved resolution criterion is applied in a dual-mesh hybrid LES/RANS solver. Numerical simulations with the hybrid solver suggest that the interactions between the length-scale resolution indicator and the solution are moderate, and that favorable comparisons with benchmark results are obtained. In summary, we demonstrate that the modified length-scale based resolution indicator performs satisfactorily in both pure LES and hybrid simulations. Therefore, it is selected as a promising candidate to provide reliable predictions of resolution adequacy for individual cells in hybrid LES/RANS simulations.
\end{abstract}

Keywords Mesh resolution · Hybrid LES/RANS methods · Turbulence modeling

H. Xiao $(\bowtie) \cdot$ J. Wang

Department of Aerospace and Ocean Engineering, Virginia Tech, Blacksburg, VA, 24060, USA

e-mail: hengxiao@vt.edu

P. Jenny $(\bowtie)$

Institute of Fluid Dynamics, ETH Zürich, 8092, Zurich, Switzerland

e-mail: jenny@ifd.mavt.ethz.ch 


\section{Introduction}

Large-Eddy Simulation (LES) has been very successful for free-shear flows. However, the high computational cost for wall-bounded flows is still a major hurdle for the application of LES in industrial and natural flow studies. Many hybrid methods have been developed to overcome this difficulty, where RANS (Reynolds-Averaged Navier-Stokes) equations are solved in the near-wall region and LES is performed in the free-shear region away from the wall. This is to avoid excessive mesh requirement and the associated computational cost $\left(\sim R e^{1.8}\right)$ of properly resolving the wall in LES [1].

In many of the hybrid methods LES and RANS regions are separated by clearly defined interfaces (either solution-dependent or predefined according to wall distances). In the hybrid model recently proposed by Xiao and Jenny [2], each cell is assigned to either the LES or the RANS region according to the resolution adequacy of this cell. Although not prescribed by the formulation, a physically sound evaluation procedure leads to clearly separated LES and RANS regions and not randomly interlaced LES and RANS cells. In all these hybrid methods, a major question is how to evaluate the resolution locally and dynamically during the simulations.

The evaluation of mesh resolution in this context is rather unique, which is different from that in the context of quality control and reliability of LES in industrial simulations. Resolution evaluation in the latter scenarios has long been a topic of research in various application areas (see, e.g., [3-6]). In these cases, one usually performs simulations on a mesh designed according to established best-practice-guides (e.g., [7]), and then assesses some integral quantities or performs another simulation on a refined mesh in order to determine whether to accept or reject the simulation results. However, in the context of hybrid LES/RANS methods, the objective is to evaluate resolution adequacy of each cell to determine whether to assign it to the LES or to the RANS regions. Consequently, the evaluation needs to be conducted on the fly based on the current solution, which makes it even more challenging than the evaluation in the context of quality control of LES as mentioned above.

In the literature many criteria or indicators to evaluate grid resolutions in LES have been proposed and used, including the following notable examples:

(1) indication based on the percentage of resolved turbulent quantities including turbulent kinetic energy (TKE) $k$, dissipation rate $\varepsilon$, and shear stresses (see, e.g., [8, 9]);

(2) indication based on the energy spectrum (compared to a model spectrum); and

(3) indication based on the ratio between the turbulent length scales and grid spacing, with the former being estimated from turbulent kinetic energy and dissipation rate.

For conciseness, hereafter they are referred to as resolved-percentage criteria, energy spectrum criteria and length-scale criteria, respectively.

Davidson conducted extensive studies on the evaluation of appropriate resolution for LES of simple wall-bounded flows [9] and recirculating flows [10]. Results obtained with the resolved percentage of turbulent quantities $(k$ and $\varepsilon$ ) criterion and the energy spectrum criterion ( 1 and 2 above) were compared with those evaluated from two-point correlations, which were regarded as the reliable benchmark criterion in his studies. It was concluded that none of the evaluated criteria other than the benchmark one gave reliable results consistently. In particular, for the criteria based on resolved turbulent quantities, the difficulty is the lack of a consistent threshold value, above which the resolution can be regarded adequate. In the literature these criteria have mostly been taken for granted, or at least the issue was not considered significant enough to warrant separate investigations. A main conclusion from 
Davidson's studies was that the two-point correlation is the most and only reliable criterion for resolution evaluation. It was further interpreted that two-point correlations indicate the number of cells used to resolve the turbulent structure in each direction, and thus the user can judge the sufficiency of the resolution depending on the purpose of the simulations.

Unfortunately, despite its convenient physical interpretation, the two-point criterion (as well as the energy spectrum criterion) is not convenient for implementations in hybrid LES/RANS codes, where the evaluation needs to be performed on the fly and ideally should be employed for individual cells. It is noted that the length-scale criterion (item 3 above) is based on the ratio between turbulent length scales and cell sizes, and has similar interpretations as the two-point correlation criterion. Since all the information needed for the length-scale criterion is local to each cell, it can be much more easily implemented, and therefore it is natural to ask how consistent the two criteria are. Although the "turbulent length "scales" implied by two-point correlations have different meaning than those estimated from turbulent kinetic energy and dissipation rate, theoretically the two length scales should be of the same order (see, e.g., [11], Chapter 6). If the lengths-scale criterion correlates well with the two-point criterion, the former is certainly preferred in dynamic evaluation in hybrid simulations due to the convenience of implementation. Another difficulty that is unique for the resolution evaluation in hybrid LES/RANS simulations is the possible inter-dependence and interactions with the solutions. Specifically, the resolution indicators are evaluated based on the results obtained during the simulations; on the other hand, the resolution evaluation would determine the division into LES and RANS regions and thus influences the simulation results. To address these issues, we studied the length scale criterion using a similar approach as adopted by Davidson [9]. However, a major difference that distinguishes the present work from previous research $[4,5,9,10]$ is that our study focuses on evaluating the resolution for determining the LES/RANS interfaces in hybrid simulations. Note that while Davidson [9] used a hybrid LES/RANS solver in his study, that was to allow for simulating wall-bounded flows at high Reynolds numbers with reasonable computational cost. The purpose of his studies was still in the context of quality control and reliability assessment of LES, and not to determine LES and RANS regions in hybrid simulations. Breuer et al. [12] proposed and investigated a few criteria for dynamic resolution evaluation in hybrid LES/RANS simulations. However, several of the formulations are specific to their particular hybrid framework, and wall-distances are involved in these criteria, which is not desirable considering that for simulations in complex geometries wall-distances may not be well-defined.

Even though no criterion may be completely satisfactory, based on the way the indicators are used in hybrid simulations, we argue that a good resolution indicator should ideally:

(1) change (increase) monotonically in response to mesh refinements;

(2) have reasonable correlation with the actual resolution;

(3) be relatively insensitive on constants in the subgrid-scale (SGS) models.

For conciseness hereafter these three resolution indicator requirements are referred to as positive responses, faithful representation, and parametric insensitivity requirements. While it is straightforward to validate against the requirements of positive response and parametric insensitivity, the faithful representation requirement is more difficult to check. Even for cases where benchmark Direct Numerical Simulation (DNS) results are available, only global quantities can be compared to the benchmark data and this does not directly reveal the adequacy of mesh resolution in each region or each cell. Moreover, there are many factors other than mesh resolution (among them are numerics and turbulence models) that 
could influence the agreement with benchmark results, and errors from different sources may cancel each other. Therefore, we used a two-point correlation indicator as reference, because it is physically justified and has a similar interpretation as the length-scale criterion. In absence of better alternatives, the two-point correlation criterion is a reasonable choice. Although theoretically the two-point correlation criterion is closely related to other criteria (e.g., resolved TKE percentage, length-scale ratio) [11], it is rarely used in the literature as a method to directly evaluate LES resolution. The work of Davidson [9] is the only such study we are aware of. For this reason, this study also attempts to put the two-point correlation as a resolution indicator under physical scrutiny. In this paper, we constrain our discussions to the resolution evaluation of LES with SGS models and implicit filtering. LES with explicit filtering (with additional meshes for the filtering) and Monotonically Integrated LES (without explicit SGS model) will introduce additional complexities to the study, and are outside the scope of the current work. RANS equations in the context of most hybrid LES/RANS studies (this one included) are unsteady, and thus they are more appropriately referred to as URANS. However, in the literature they are sometimes referred to as RANS for simplicity. Without causing confusion, this convention of terminology shall be followed here. The rest of the paper is organized as follows. In Section 2, details of the methodology of resolution evaluation, the solver, and the numerical methods are presented. The modified version of the length-scale resolution indicator is motivated and discussed. In Section 3, the resolution indicators based on turbulent length scales and two-point correlations are evaluated in LES and the two indicators are compared. The sensitivity of the resolution indicators on the model constant is investigated along with several other criteria. In Section 4, the resolution indicator is tested in a hybrid LES/RANS solver to observe the interactions with the solutions and to compare with benchmark results. Several open issues are discussed in Section 5. Finally, Section 6 concludes the paper.

\section{Solvers and Methodology}

\subsection{Numerical methods and solvers}

In this study, both LES and hybrid LES/RANS solvers were used, both of which were developed based on the open-source CFD platform OpenFOAM [13]. Aspects that are common to both solvers are introduced here. The hybrid solver is briefly introduced in Section 4. More details specific to the hybrid solver and numerical examples can be found in refs. [2] and [14]. The continuity and momentum equations for incompressible turbulent flows were solved using the PISO (Pressure Implicit with Splitting of Operators) algorithm on an unstructured mesh [15]. Collocated grids were used and the Rhie and Chow interpolation was used to prevent the pressure-velocity decoupling [16]. Spatial derivatives were discretized with the finite volume method using the secondorder central scheme for both convection and diffusion terms. A second-order implicit time-integration scheme was used to discretize the temporal derivatives. For SGS modeling, a one-equation eddy (OEE) viscosity model was employed [17] with a standard choice of model coefficients, i.e., $C_{k}=0.094$ and $C_{\varepsilon}=1.048$. When the turbulence production equals dissipation, the OEE model is equivalent to the Smagorinsky SGS model with constant $C_{s}=C_{k}^{3 / 4} / C_{\varepsilon}^{1 / 4}$ [17]. Therefore, the choice of $C_{k}$ and $C_{\varepsilon}$ here corresponds to $C_{s}=0.168$, which approximately corresponds to the standard value for the Smagorinsky model. 


\subsection{Evaluated resolution criteria}

As discussed in Section 1, the resolution criterion based on the ratio of turbulent length scale and grid spacing is defined as follows:

$$
\phi_{l}=\frac{l_{t}}{\Delta}
$$

where the grid spacing $\Delta$ is usually defined as $\max (\Delta x, \Delta y, \Delta z)$ or as $V^{1 / 3}$, where $V$ is the cell volume; $\Delta x, \Delta y$, and $\Delta z$ (also denoted as $\Delta x_{1}, \Delta x_{2}$, and $\Delta x_{3}$ later on) are the cell dimensions in $x$-, $y$-, and $z$-directions, respectively. The turbulent length scale $l_{t}$ is estimated based on the turbulent kinetic energy $k$ and the dissipation rate $\varepsilon$ as

$$
l_{t}=\frac{k^{3 / 2}}{\varepsilon}
$$

with

$$
k=\frac{1}{2}\left\langle u_{i}^{\prime \prime} u_{i}^{\prime \prime}\right\rangle^{\mathrm{AVG}}+k^{\mathrm{sgs}}
$$

where $k$ is the total TKE including the resolved part and the SGS part; $u_{i}^{\prime \prime}$ is the resolved velocity fluctuation in the $i^{\text {th }}$ direction (see Eq. (18) for specific definition); $\varepsilon$ is the total dissipation including the resolved and the SGS parts (see Eq. (17c)); $\left\langle u_{i}^{\prime \prime} u_{j}^{\prime \prime}\right\rangle$ is the resolved turbulent stresses, $k^{\mathrm{sgs}}$ is the SGS turbulent kinetic energy. Note that the operator $\langle\cdot\rangle^{A V G}$ in this work is more appropriately interpreted as time averaging (for statistically stationary flows) or low-pass filtering (for non-stationary flows; see Eq. (17) for definition) since they operate on LES quantities. In a typical hybrid LES/RANS framework $\phi_{l}$ is computed for all cells in the field. For each cell, if $\phi_{l}$ in this cell is larger than a specified threshold value $\phi_{0}$, it is considered well-resolved and is classified for the LES region; otherwise it is considered under-resolved and is classified for the RANS region. An alternative is to pre-specify the interface between LES and RANS regions, evaluate the resolution near the interfaces and then adjust the interface locations accordingly during the simulations (e.g., [18]). It is also possible to combine the resolution indicators and wall-distance to determine LES/RANS interfaces as in ref. [12]. The spatial structures of the turbulent flow velocity field can be characterized by the two-point correlation $R_{i j}$ defined as

$$
R_{i j}\left(\mathbf{x}_{0}, \mathbf{x}, t\right)=\left\langle u_{i}^{\prime \prime}\left(\mathbf{x}_{0}, t\right) u_{j}^{\prime \prime}\left(\mathbf{x}_{0}+\mathbf{x}, t\right)\right\rangle
$$

where $\mathbf{x}_{0}$ and $t$ are spatial and temporal coordinates, $\mathbf{x}$ is the offset vector between the two points, $u_{i}$ is the fluctuation of the $i^{t h}$ velocity component, and $\langle\cdot\rangle$ indicates statistical averaging. As explained by Davidson [9], the two-point correlation indicates the size of turbulent eddies in the corresponding direction, from which one can determine the number of cells used to resolve these eddies. The length-scale criterion with the resolution indicator $\phi_{l}$ defined as a scalar field in Eq. (1) is not fully consistent with the interpretation of the two-point correlation criterion. Since the eddies are usually stretched and anisotropic, particularly near the wall, the scalar $l_{t}$ is not able to fully characterize the eddy size. Dividing $l_{t}$ by $V^{1 / 3}$ or $\max (\Delta x, \Delta y, \Delta z)$ makes the physical meaning even more ambiguous. To overcome this inconsistency, one can modify the above formulation and define the turbulent eddy size by the following tensor:

$$
\mathcal{L}_{i j}=\frac{1}{\varepsilon}\left[\frac{3}{2}\left\langle u_{i}^{\prime \prime} u_{j}^{\prime \prime}\right\rangle+k^{\mathrm{sgs}} \delta_{i j}\right]^{3 / 2},
$$

where $\delta_{i j}$ is the Kronecker delta, and other variables are defined in the same way as in Eq. (3). The Reynolds stress field, including the resolved part and the contribution from 
the SGS kinetic energy, is used to characterize the eddy sizes. The scalar dissipation rate $\varepsilon$ is used in the formulation since most of the dissipation occurs at the sub-grid scale where anisotropy is not important. The same argument holds for distributing $k^{\text {sgs }}$ equally among three directions, since the turbulent kinetic energy at the sub-grid scale has no directional preference except very close to the walls. As the Reynolds stress tensor is symmetric, it has three orthogonal eigenvectors which, after normalization, would form a set of orthonormal basis, represented by unit vectors $\left(\hat{\mathbf{e}}_{1}, \hat{\mathbf{e}}_{2}, \hat{\mathbf{e}}_{3}\right)$. The corresponding real eigenvalues, denoted as $\lambda_{1}, \lambda_{2}$, and $\lambda_{3}$, can be considered as the dimensions of the large eddies in the three eigendirections. On the other hand, a vector $\Delta$ characterizing the shape of the cell (the longest diagonal of the cell was used in this study) can be projected onto the basis $\hat{\mathbf{e}}_{i}$ to obtain the cell dimensions, that is,

$$
\boldsymbol{\Delta}=\Delta x_{1} \hat{\mathbf{e}}_{1}+\Delta x_{2} \hat{\mathbf{e}}_{2}+\Delta x_{3} \hat{\mathbf{e}}_{3},
$$

or equivalently

$$
\Delta x_{1}=\boldsymbol{\Delta} \cdot \hat{\mathbf{e}}_{1}, \quad \Delta x_{2}=\boldsymbol{\Delta} \cdot \hat{\mathbf{e}}_{2}, \quad \text { and } \quad \Delta x_{3}=\boldsymbol{\Delta} \cdot \hat{\mathbf{e}}_{3},
$$

where $\Delta x_{1}, \Delta x_{2}, \Delta x_{3}$ can be regarded as the generalized cell dimensions in the three Cartesian coordinate axes for a polyhedron. The definition of $\Delta_{i}$ is illustrated in Fig. 1a for a two-dimensional quadrilateral with the vector $\Delta$ and its projections on the two coordinate axes $\hat{\mathbf{e}}_{1}$ and $\hat{\mathbf{e}}_{2}$ shown as arrows. In the trivial case of a structural grid with hexahedral cells whose edges are aligned with coordinate axes, $\Delta x_{i}$ is simply the cell size in the $i^{\text {th }}$ direction. Theoretically, this definition is also applicable to tetrahedral and triangular cells, as shown in Fig. 1b. Admittedly, however, unstructured meshes with tetrahedral cells were not tested in this work, and thus further investigations are needed

With the definition of cell length scales, the resolution indication vector based on the Reynolds stress tensor and the cell sizes can be defined as:

$$
\phi_{i}^{(l)}=\frac{\lambda_{i}}{\Delta x_{i}} \quad \text { (no summation on repeated indexes). }
$$

The modified formulation of turbulent length scale based on $\mathcal{L}_{i j}$ is frameindependent. However, in this study we adapted a simpler approach and used instead

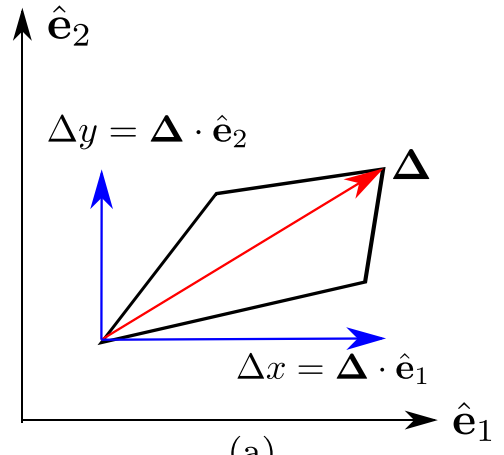

(a)

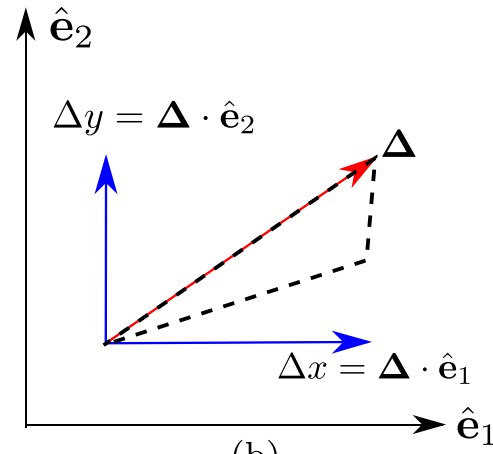

(b)

Fig. 1 Definition of length scales in each coordinate direction for (a) quadrilateral cells and (b) triangular cells 
the diagonal components of the Reynolds stress tensor to characterize the eddy sizes:

$$
\mathbf{l}_{t}=\left[\frac{\left(\frac{3}{2}\left\langle u^{\prime \prime} u^{\prime \prime}\right\rangle+k^{\mathrm{sgs}}\right)^{3 / 2}}{\varepsilon}, \frac{\left(\frac{3}{2}\left\langle v^{\prime \prime} v^{\prime \prime}\right\rangle+k^{\mathrm{sgs}}\right)^{3 / 2}}{\varepsilon}, \frac{\left(\frac{3}{2}\left\langle w^{\prime \prime} w^{\prime \prime}\right\rangle+k^{\mathrm{sgs}}\right)^{3 / 2}}{\varepsilon}\right]^{T} .
$$

The resolution indicator based on the component-wise ratios of $\mathbf{l}_{t}$ and $\Delta$ is thus defined accordingly as follows:

$$
\boldsymbol{\phi}^{(l)}=\left[\frac{\left(\frac{3}{2}\left\langle u^{\prime \prime} u^{\prime \prime}\right\rangle+k^{\mathrm{sgs}}\right)^{3 / 2}}{\varepsilon \Delta x}, \frac{\left(\frac{3}{2}\left\langle v^{\prime \prime} v^{\prime \prime}\right\rangle+k^{\mathrm{sgs}}\right)^{3 / 2}}{\varepsilon \Delta y}, \frac{\left(\frac{3}{2}\left\langle w^{\prime \prime} w^{\prime \prime}\right\rangle+k^{\mathrm{sgs}}\right)^{3 / 2}}{\varepsilon \Delta z}\right]^{T}
$$

with the three components of $\phi^{(l)}$ denoted as $\phi_{x}^{(l)}, \phi_{y}^{(l)}$, and $\phi_{z}^{(l)}$, respectively. With this simplified formulation, the definition of $\phi^{(l)}$ is frame-dependent and thus it is less rigorous mathematically. It is not exactly equivalent to the original formulation in Eqs. (5)-(8). However, it offers the convenience of validating against existing physical insights in canonical flows, as will be illustrated in Section 3. This issue is further discussed in Section 5.2.

Similar to the original scalar form length-scale formulation in Eqs. (1) and (2), the resolution based on $\phi^{(l)}$ is obtained by comparing either $\phi_{\min }^{(l)} \equiv \min \left(\phi_{x}^{(l)}, \phi_{y}^{(l)}, \phi_{z}^{(l)}\right)$ or the Euclidean norm $\left\|\phi^{(l)}\right\|$ with a threshold value. It is expected that different threshold values are needed depending on whether $\phi_{\min }^{(l)}$ or $\left\|\phi^{(l)}\right\|$ is used. In this study, when the resolution adequacy of cells must be evaluated, $\phi_{\min }^{(l)}$ is compared to a specified threshold value $\phi_{0}$. One may also compare $\left\|\phi^{(l)}\right\|$ with a specified vector $\phi_{0}$ component-wise, because theoretically it can be justified to require different relative resolutions in each direction. This approach would, however, introduce more ad hoc parameters into the formulation. It will preliminarily be explored in Section 4. Due to the uncertainties in choosing the proper functional form of $\phi^{(l)}$ to conduct comparisons, whenever possible we focus on the streamwise and spanwise components $\left(\phi_{x}^{(l)}\right.$ and $\left.\phi_{z}^{(l)}\right)$ instead. This is due to the difficulty caused by the lack of homogeneity in the wall-norm $y$ direction. Also note that the study by Davidson [9] did not consider wall-normal resolution. Instead, they used a hybrid LES/RANS method to circumvent this difficulty. In this study, the two-point correlation $\rho_{v v}$ and the resolution indicator $\phi_{y}^{(l)}$ in the wall normal direction are studied in one computational case presented in Section 3.1.3.

The two-point correlation criterion is based on the correlation coefficient $\rho_{i j}$ of velocities at two points, which is obtained by normalizing $R_{i j}$ as defined in Eq. (4) by the root mean square (rms) of the three velocity components. The correlation coefficient at a specific point $\mathbf{x}_{\mathbf{0}}$ is a third-order tensor which depends on the offset and the two correlation velocities. In this study the emphasis was placed on the following components:

$$
\begin{gathered}
\rho_{\mathrm{uu}}\left(\mathbf{x}_{0}, x\right)=\frac{\left\langle u^{\prime \prime}\left(\mathbf{x}_{0}\right) u^{\prime \prime}\left(\mathbf{x}_{0}+x \hat{\mathbf{e}}_{1}\right)\right\rangle}{u_{\mathrm{rms}}\left(\mathbf{x}_{0}\right) u_{\mathrm{rms}}\left(\mathbf{x}_{0}+x \hat{\mathbf{e}}_{1}\right)}, \\
\rho_{\mathrm{vv}}\left(\mathbf{x}_{0}, y\right)=\frac{\left\langle v^{\prime \prime}\left(\mathbf{x}_{0}\right) v^{\prime \prime}\left(\mathbf{x}_{0}+y \hat{\mathbf{e}}_{2}\right)\right\rangle}{v_{\mathrm{rms}}\left(\mathbf{x}_{0}\right) v_{\mathrm{rms}}\left(\mathbf{x}_{0}+y \hat{\mathbf{e}}_{2}\right)}, \text { and } \\
\rho_{\mathrm{ww}}\left(\mathbf{x}_{0}, z\right)=\frac{\left\langle w^{\prime \prime}\left(\mathbf{x}_{0}\right) w^{\prime \prime}\left(\mathbf{x}_{0}+z \hat{\mathbf{e}}_{3}\right)\right\rangle}{w_{\mathrm{rms}}\left(X_{0}\right) w_{\mathrm{rms}}\left(\mathbf{x}_{0}+z \hat{\mathbf{e}}_{3}\right)},
\end{gathered}
$$


where $u^{\prime \prime}, v^{\prime \prime}$ and $w^{\prime \prime}$ denote velocity fluctuations in $x$-, $y$-, and $z$-directions, respectively; $\mathbf{x}_{0}$ is the base point coordinate; $x \hat{\mathbf{e}}_{1}, y \hat{\mathbf{e}}_{2}$ and $z \hat{\mathbf{e}}_{3}$ are the offset distances with respect to the base point in the streamwise, wall-normal, and spanwise directions, respectively. The location of base point $\mathbf{x}_{0}$ is specified for individual cases when necessary. According to Davidson [9], the two-point correlation can be interpreted as follows: the distance over which $\rho$ drops below 0.3 is considered as the size of the "eddy" in this direction. The number of cells in this distance indicates the number of cells used to resolve this "eddy". This interpretation is more convenient than the "integral length scale" defined as the area under the correlation curve, since no integration is needed. Although the cutoff correlation 0.3 seems arbitrary and the interpretation may not be physically rigorous, it rarely caused ambiguity in practice. This observation also supports the approach adopted by Davidson [9].

\subsection{Methodology of assessing criteria}

We evaluated resolution criteria for two cases: plane channel flow at $R e_{\tau}=4000$ (based on friction velocity $u_{*}$ and half channel width $\delta$ ) and the flow over periodic hills at $R e=10595$ (based on hill height $H$ and bulk velocity $U_{b}$ over the hill crest), representing simple flows and complex flows with recirculations, respectively. For both cases, a pressure gradient was applied in the entire domain to enforce the desired constant mass flux. Simulations were conducted on five meshes for both cases: a baseline mesh, two meshes refined in streamwise $(x)$ direction by a factor of two (denoted as mesh A; $0.5 \Delta x$ ) and four (mesh $\mathrm{B} ; 0.25 \Delta x$ ) with respect to the baseline mesh, and two meshes refined in spanwise (z) direction by a factor of two (mesh $\mathrm{C} ; 0.5 \Delta z$ ) and four (mesh D; $0.25 \Delta z$ ). For the plane channel flow, we investigated two additional meshes, which were obtained by refining the baseline mesh in both $x$ and $z$ directions by a factor of 2 (mesh E; $0.5 \Delta x \times 0.5 \Delta z$ ), and in the wall-normal $(y)$ direction by a factor of two (mesh F; 0.5 $\Delta y$ ), respectively. The number of cells and grid spacing for all the meshes will be presented separately for each case in Tables 1 and 4.

\section{Resolution Indicators in LES}

In this section, standalone LES results are presented for the two cases: plane channel flow at $R e_{\tau}=4000$ and flow over periodic hills at $R e=10595$. The setups of each case are explained and the resolution indicators evaluated from two-point correlations and lengthscale ratios are compared and discussed. Finally, the influence of model constants on the

Table 1 Computational mesh and resolution in wall units for plane channel flow of $R e_{\tau}=4000$

\begin{tabular}{llllll}
\hline mesh & $\mathrm{N}_{\mathrm{x}}$ & $\mathrm{N}_{\mathrm{y}}$ & $\mathrm{N}_{\mathrm{z}}$ & $\Delta x^{+}$ & $\Delta z^{+}$ \\
\hline baseline & 70 & 140 & 130 & 400 & 100 \\
$0.5 \Delta x(\mathrm{~A})$ & 140 & 140 & 130 & 200 & 100 \\
$0.5 \Delta z(\mathrm{~B})$ & 70 & 140 & 260 & 400 & 50 \\
$0.25 \Delta x(\mathrm{C})$ & 280 & 140 & 130 & 100 & 100 \\
$0.25 \Delta z(\mathrm{D})$ & 70 & 140 & 520 & 400 & 25 \\
$0.5 \Delta x \times 0.5 \Delta z(\mathrm{E})$ & 140 & 140 & 260 & 200 & 50 \\
$0.5 \Delta y(\mathrm{~F})$ & 70 & 280 & 130 & 400 & 100 \\
\hline
\end{tabular}


length-scale resolution indicator are investigated by comparing with two other indicators based on percentage of resolved turbulent quantities.

\subsection{Plane channel flow at $R e_{\tau}=4000$}

The computational domain size was chosen as $2 \pi \times 2 \times \pi$ in streamwise $(x)$, wall-normal $(y)$, and spanwise $(z)$ directions. All the length scales in this case are normalized by the half channel width $\delta$. The number of cells in $x$ - and $z$-directions $\left(N_{x}\right.$ and $\left.N_{z}\right)$ and the cell spacing $\left(\Delta x^{+}\right.$and $\left.\Delta z^{+}\right)$in viscous units are presented in Table 1 . The number of cells in $y$-direction is $N_{y}=140$ with cell sizes in viscous units ranging from $\Delta y_{\min }^{+}=7.6$ (the first cell from the wall) to $\Delta y_{\max }^{+}=190$ (the cell at the channel center). Cases A-E and the baseline case all have the same $N_{y}, \Delta y_{\min }^{+}$, and $\Delta y_{\max }^{+}$. The simulations were conducted for 50 through-times to obtain converged statistics.

\subsubsection{Resolution implied by two-point correlations in $x$ - and $z$-directions}

The two-point correlations $\rho_{u u}(x)$ and $\rho_{w w}(z)$ were computed using velocity time-series arranged within six planes parallel to the wall. We only present results at two representative locations, i.e., at $y_{0}=0.055$ (located in the near wall region with $y^{+} \equiv y / y^{*}=220$, where $y^{*}=v / u_{\tau}$ is the viscous unit, and $u_{\tau}$ is the friction velocity) and $y_{0}=0.44$ (located in the free-shear region with $y^{+}=1760$ ) in Fig. 2. Results on other planes show the same qualitative features as discussed here.

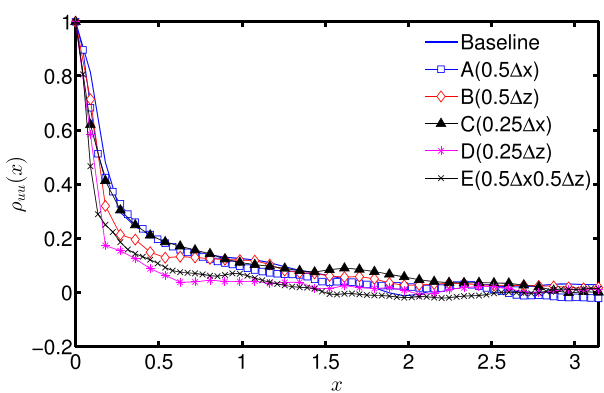

(a)

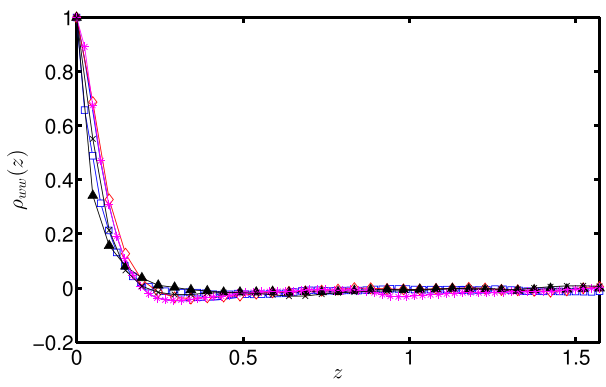

(c)

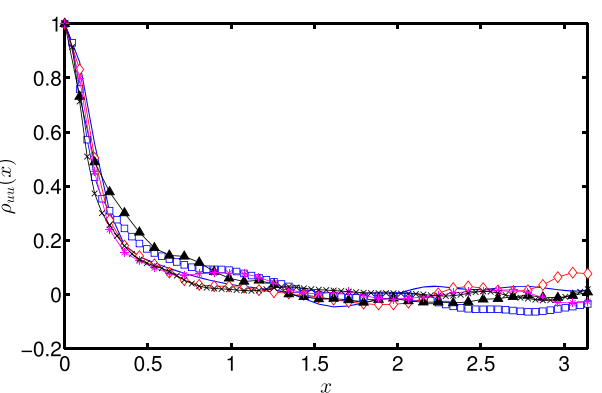

(b)

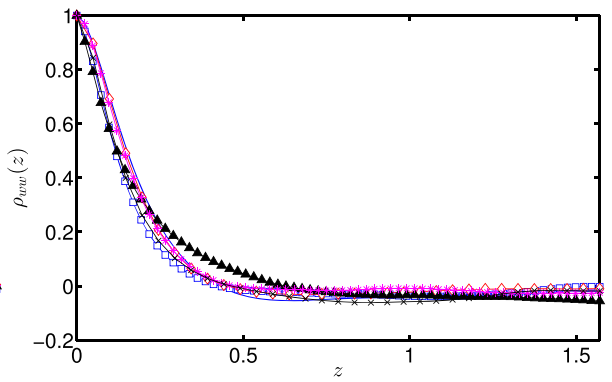

(d)

Fig. 2 Two-point correlations for plane channel flow at $R e_{\tau}=4000$. Top: streamwise correlations $\rho_{\text {uu }}(x)$ at the two wall-normal locations $y_{0}=0.055$ (plot a) and $y_{0}=0.44$ (plot b). Bottom: spanwise correlations $\rho_{\mathrm{ww}}(z)$ at the two wall-normal locations $y_{0}=0.055$ (plot c) and $y_{0}=0.44$ (plot d) 
The general observation from Fig. 2 is that the two-point correlations computed from different meshes are similar overall, particularly in the regions where significant correlations exist $(\rho>0.3)$. From the overall agreement, we infer the following: (1) all the meshes (baseline and A-E) are able to represent at least the basic eddy structures of the flow, and no major artificial correlations were observed; and (2) the two-point correlations can be considered as approximate indications of the characteristic eddy sizes. Minor deviations in the plots will be analyzed later. With these two assumptions one can proceed and identify the "eddy" sizes ( $l_{x}$ and $l_{z}$ in streamwise and spanwise directions, respectively) of the flows. This was done by measuring the distance over which the correlation drops below 0.3 (i.e., identifying the intersections between the horizontal line $\rho=0.3$ and the $\rho_{\text {uu }}(x)$ or the $\rho_{\mathrm{ww}}(z)$ curves). Through this procedure it was identified that $l_{x}=0.3$ at $y_{0}=0.055$ from Fig. 2a and $l_{x}=0.26$ at $y=0.44$ from Fig. $2 \mathrm{~b}$, where results obtained from mesh A $(0.5 \Delta x)$ were used. Similarly, it was found that $l_{z}=0.1$ at $y_{0}=0.055$ and $l_{z}=0.22$ at $y=0.44$, using results from mesh $\mathrm{B}(0.5 \Delta z)$. In summary, the two-point correlation data suggest that the horizontal extent of the "eddies" at $y_{0}=0.055$ is $l_{x} \times l_{z}=0.3 \times 0.1$, indicating elongated turbulent structures; and that at $y_{0}=0.44$ it is $l_{x} \times l_{z}=0.26 \times 0.22$, representing relatively isotropic structures. This observation is qualitatively consistent with the well-known results obtained from DNS of channel flows (see, e.g., ref. [19] and Chapter 7 of ref. [11]). One can argue that correlation data obtained from different meshes may not accurately indicate eddy structures, and that Case E with mesh $0.5 \Delta x \times 0.5 \Delta z$ would be a more appropriate case to estimate the eddy sizes. It can be seen that with Case E the observation would still be valid, although the exact numbers would be slightly different.

Furthermore we can measure the number of cells over which the correlations drop below 0.3 as indication of the resolution in this direction (i.e., $l_{x} / \Delta x$ ), which are presented in Table 2 for $x$ direction and in Table 3 for $z$ direction. The cases in Table 2 are grouped according to $N_{x}$ and those in Table 3 according to $N_{z}$. If the two-point correlations in all cases had implied identical eddy size, the cases in the same group should give the same resolution, since they have the same grid spacing. This is generally consistent with what is observed in Tables 2 and 3, with minor deviations at $y_{0}=0.055$. Another implication is that the second group should approximately lead to resolution indicators twice as large as the first group, and the third group four times. This expectation is also met by the data presented in Tables 2 and 3. Again, some minor deviations come from meshes at $y_{0}=0.055$. A possible explanation is discussed below.

Although the two-point correlations obtained from different meshes agree with each other overall, minor deviations are observed in most of the plots except Fig. 2d. Deviations as measured by the relative differences of implied eddy sizes are more obvious for Fig. 2a

Table 2 Streamwise $(x)$ resolution as indicated by the number of cells used to resolve the eddy structures in $x$ direction. The numbers were obtained from streamwise two-point correlations $\rho_{\mathrm{uu}}(x)$ presented in Fig. 2. Results are presented for the two wall-normal locations $y_{0}=0.055$ and $y_{0}=0.44$

\begin{tabular}{llll}
\hline mesh & $N_{x}$ & $y_{0}=0.055$ & $y_{0}=0.44$ \\
\hline baseline & 70 & 3 & 3 \\
$\mathrm{~B}(0.5 \Delta z)$ & 70 & 2.3 & 2.9 \\
$\mathrm{D}(0.25 \Delta z)$ & 70 & 2 & 2.8 \\
$\mathrm{~A}(0.5 \Delta x)$ & 140 & 6.5 & 6.1 \\
$\mathrm{E}(0.5 \Delta x \times 0.5 \Delta z)$ & 140 & 3.5 & 5 \\
$\mathrm{C}(0.25 \Delta x)$ & 280 & 12.5 & 15.2 \\
\hline
\end{tabular}


Table 3 Spanwise ( $z$ ) resolution as indicated by the number of cells used to resolve the eddy structures in $z$ direction. The numbers were obtained from spanwise two-point correlations $\rho_{\mathrm{ww}}(r)$ in Fig. 2. Results are presented for the two wall-normal locations $y_{0}=0.055$ and $y_{0}=0.44$

\begin{tabular}{llll}
\hline mesh & $N_{z}$ & $y_{0}=0.055$ & $y_{0}=0.44$ \\
\hline baseline & 130 & 4 & 8.2 \\
A $(0.5 \Delta x)$ & 130 & 3 & 8 \\
$\mathrm{C}(0.25 \Delta x)$ & 130 & 2.6 & 8.1 \\
$\mathrm{~B}(0.5 \Delta z)$ & 260 & 8.2 & 16 \\
$\mathrm{E}(0.5 \Delta x \times 0.5 \Delta z)$ & 260 & 7.9 & 16.5 \\
$\mathrm{D}(0.25 \Delta z)$ & 520 & 16 & 33 \\
\hline
\end{tabular}

and $\mathrm{c}$ for $y_{0}=0.055$ than those for (b) and (d) for $y_{0}=0.44$. This seems to suggest that the resolution at $y_{0}=0.44$ is better than that at $y_{0}=0.055$. Considering that $\Delta x$ and $\Delta z$ are uniform across the domain, we could infer that the eddy sizes at $y_{0}=0.055$ is smaller. As we know, this is only true for the spanwise dimension $l_{z}$ but not for the streamwise dimension $l_{x}$. Throughout this study, the same paradox has occasionally been observed in other cases with meshes $\mathrm{C}(0.25 \Delta x)$ and $\mathrm{D}(0.25 \Delta z)$. Since the baseline mesh was designed to have a reasonable cell aspect ratio $(\Delta x / \Delta z=4)$ to account for the elongation of the near-wall turbulent structures, the meshes $\mathrm{C}$ and $\mathrm{D}$ obtained by refining the baseline mesh by a factor of four in one direction only ( $x$ or $z$ ) have led to inappropriate aspect ratios ( $\Delta x / \Delta z=1$ and 16 , respectively). This may be the reason for spurious correlations in some cases leading to deviations of two-point correlation results.

\subsubsection{Resolution indicator based on turbulent length-scales in $x$-and $z$-directions}

To assess the length-scale resolution indicators against the requirements of positive response and faithful representation as discussed in Section 1, we computed the resolution indicator fields $\phi^{(l)}$ at each time-step according to Eq. (10). Temporal, streamwise, and spanwise averaging were performed on the fields to estimate the mean profiles of $\phi_{x}^{(l)}, \phi_{y}^{(l)}, \phi_{z}^{(l)}$, and $\phi_{\min }^{(l)}$ along the wall-normal direction, which is the only statistically non-homogeneous direction in this flow. These profiles are presented in Fig. 3.

The positive response requirement suggests that $\phi_{x}^{(l)}$ from mesh $\mathrm{A}(0.5 \Delta x)$ should be higher than that in the baseline mesh; and $\phi_{x}^{(l)}$ in mesh $\mathrm{C}(0.25 \Delta x)$ should be the highest. This is indeed the case as demonstrated in Fig. 3a. Furthermore, $\phi_{x}^{(l)}$ for mesh A $(0.5 \Delta x)$ and $\mathrm{C}(0.25 \Delta x)$ are approximately twice and four times, respectively, as large as for the baseline mesh, indicating that the turbulent length scales in all these meshes are similar. The positive response requirement would also imply that the meshes B and D should have the same $\phi_{x}^{(l)}$ as the baseline mesh because they have the same resolution in $x$ direction. This is also confirmed by the observations from the figure. In fact, the $\phi_{x}^{(l)}$ profiles from three groups corresponding to there streamwise cell sizes: the first group consists of mesh $\mathrm{C}$ (streamwise cell size $0.25 \Delta x$ ); the second group consists of meshes A and $\mathrm{E}$ (streamwise cell size $0.5 \Delta x$ ); and the third group consists of meshes $\mathrm{B}, \mathrm{D}$, and the baseline (streamwise cell size $\Delta x$ ). This observation is indicated in Fig. 3a with arrows. Similarly in Fig. 3c $\phi_{z}^{(l)}$ for mesh B $(0.5 \Delta z)$ is approximately twice as large as in the baseline case, and $\phi_{z}^{(l)}$ in mesh $\mathrm{D}(0.25 \Delta z)$ is approximately 4 times as large as in the baseline case. In addition, the baseline case has almost identical $\phi_{z}^{(l)}$ as that for meshes $\mathrm{A}(0.5 \Delta x)$ and $\mathrm{C}(0.25 \Delta z)$; meshes $\mathrm{B}$ and D have similar $\phi_{z}^{(l)}$. Similar to the profiles of $\phi_{x}^{(l)}$ in Fig. 3a, three distinct groups can be observed, which are also indicated in Fig. 3 for $\mathrm{c} \phi_{z}^{(l)}$. In summary, the resolution 


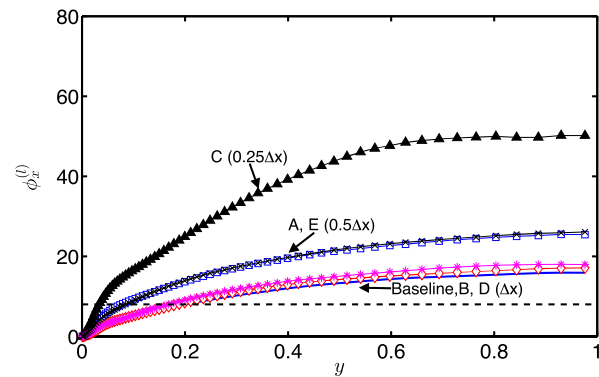

(a)

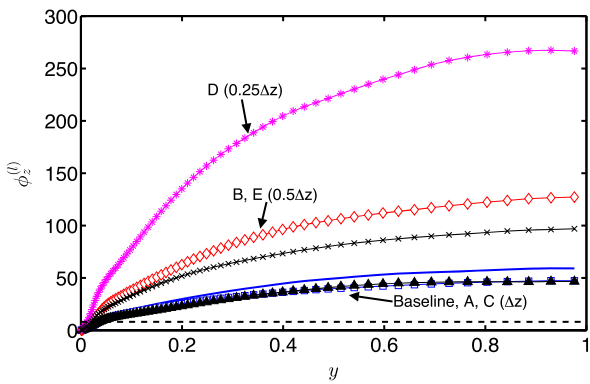

(c)

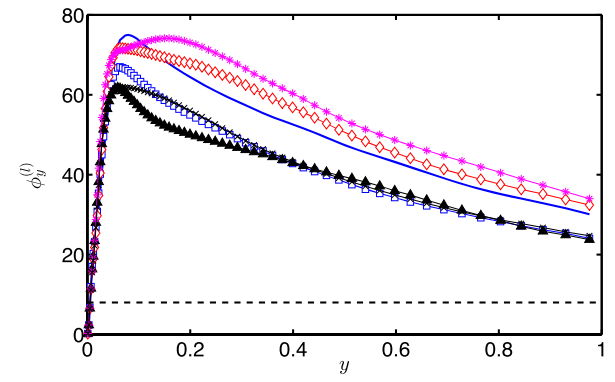

(b)

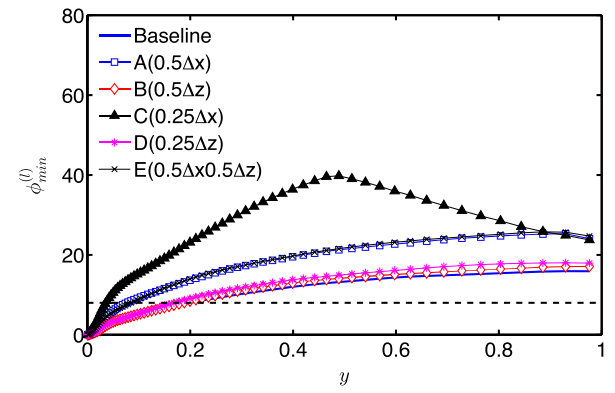

(d)

Fig. 3 Profiles of resolution indicators (a) $\phi_{x}^{(l)},(\mathbf{b}) \phi_{y}^{(l)},(\mathbf{c}) \phi_{z}^{(l)}$, and (d) $\phi_{\min }^{(l)}$ along the wall normal direction for six different meshes. Dashed lines indicate threshold value 8. In panel (a), the profiles clearly form three groups according to the streamwise $(x)$ cell sizes: mesh $\mathrm{C}$ with $0.25 \Delta x$, meshes $\mathrm{A}$ and $\mathrm{E}$ with $0.5 \Delta x$, meshes $\mathrm{B}, \mathrm{D}$, and baseline with $\Delta x$. Similar groups are identified in panel (c)

indicators $\phi_{x}^{(l)}$ and $\phi_{z}^{(l)}$ mostly show correct trends with mesh refinement. From the ratios of $\phi_{x}^{(l)}$ and $\phi_{z}^{(l)}$ for different meshes it can be inferred that the turbulent length scales $l_{t x}$ and $l_{t z}$ in $x$ and $z$ directions estimated from $k$ and $\varepsilon$ using Eq. (9) do not vary significantly for simulations with different meshes. This is consistent with the observations from the twopoint correlation criterion. One can argue that the resolution indicators $\phi_{x}^{(l)}$ and $\phi_{z}^{(l)}$ form a faithful representation of the actual resolutions in their respective directions.

As the wall-normal $(y)$ resolutions are the same for all meshes considered here (A-E), $\phi_{y}^{(l)}$ is expected to be the same as well. Admittedly, this is not exactly what we observe in Fig. 3b, particularly far away from the wall $(y>0.2)$. However, the deviations are relatively minor.

The mean profiles for $\phi_{\min }^{(l)}$ are presented in Fig. 3d. By comparing this quantity with a threshold value (e.g., $\phi_{0}=8$ ), one can determine the LES/RANS interfaces, the wall normal $y$ locations of which are indicated by the intersection between the horizontal line $\phi^{(l)}=8$ and the $\phi_{\min }^{(l)}$ curves. It can be seen that the interface is placed at approximately $y=0.2$ for the baseline case. For mesh C $(0.25 \Delta x)$ it is located at $y=0.038$, significantly closer to the wall, demonstrating that refining in $x$ direction is most effective in improving overall resolution. This is true regardless of the threshold $\phi_{0}$ value. For mesh A $(0.5 \Delta x)$, the interface is located near $y=0.1$, while for the meshes with $z$ refinement (B and $\mathrm{D}$ ) the interfaces are further away from the wall than for $\mathrm{A}$ and $\mathrm{C}$, suggesting that refining in $x$ direction is more effective for the baseline mesh. This statement also holds for any other physically reasonable values (e.g., 3-15) of $\phi_{0}$. 
The choice of the threshold value is ad hoc to some extent. In the literature, the suggested ratio of turbulent length scale $l_{t}$ and grid spacing $\Delta$ ranged between 8 and 12, depending on the precise definition of $l_{t}$ and $\Delta$ (see, e.g., [4]). Values between 3 and 12 seem acceptable in hybrid simulations according to the physical meaning of the indicator (loosely interpreted as the number of cells used to resolve the "eddies"). The analysis above suggests that the profiles of length-scale indicator $\phi^{(l)}$ are reasonable and mostly consistent with existing knowledge about this flow. In the light of interpretation of this resolution indicator as discussed above, this observation lends support to the physical relevance of the criterion. It is emphasized that the analysis here is concerned with the general behavior of the resolution indicators and thus does not depend on the choice of a particular threshold value $\phi_{0}$.

Note that the mean profile (temporal, streamwise, and spanwise averaged values) of $\phi_{\mathrm{min}}^{(l)}$ is presented and analyzed here to understand the general behavior for the length-scale resolution criteria. In actual hybrid simulations, a moving-window time-averaged value at each cell would be used to dynamically determine the resolution adequacy of a cell, and to classify the cell to the LES or RANS regions accordingly. Inevitably a time-varying and rugged LES/RANS interface would be observed. However, the average position would be consistent with those shown in Fig. 3d.

\subsubsection{Resolution indicators with wall-normal refinement}

With the methodology such as using two-point correlations as the "ground truth" to infer the adequacy of resolution of individual cells, it is difficult to study a non-homogeneous direction with grid stretch. Specifically, take for example the mesh as in Fig. 4a and the corresponding two-point correlation curve in Fig. 4b, we can see that the correlation falls below 0.3 over, say, three cells, which is probably not enough. However, due to the nonuniform grid, the message from this correlation curve would not be as clear as in the $x$ - and $z$ - directions with uniform grid-spacing, since the inadequate resolution could be attributed to the cell at the base location that is too coarse, to the large stretch ratio, or to the combination of both. Recognizing the fact that the investigation of wall-normal resolution in this study would not be as rigorous as those in streamwise and spanwise directions, we present the study of wall-normal resolution separately in this section as below.

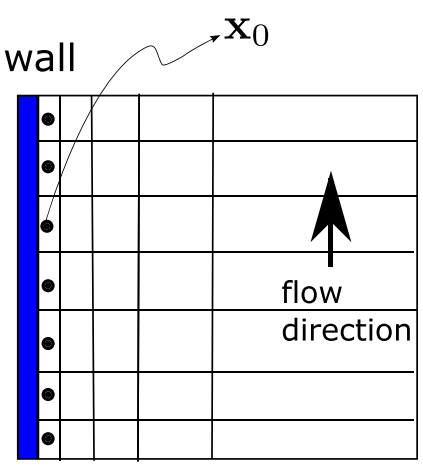

(a)

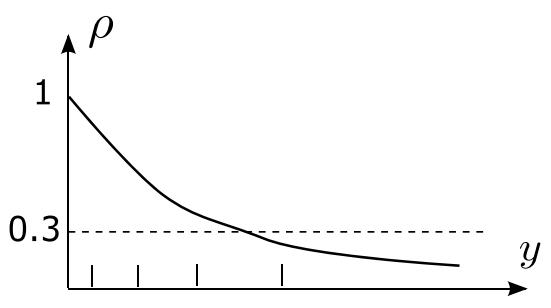

(b)

Fig. 4 (a) Mesh and (b) two-point correlation for a hypothetical wall-normal mesh resolution study. Ticks in panel (b) indicate cell boundaries 
Figure 5 displays the profiles of the two-point correlations $\rho_{v v}(y)$ and the length-scalebased resolution indicators $\phi_{y}^{(l)}$ in the wall-normal direction computed for the baseline case (with mesh spacing $\Delta y$ ) and case $\mathrm{F}$ (with mesh spacing $0.5 \Delta y$ ). The base location $\mathbf{x}_{0}$ (see Eq. (12)) is chosen to be the first cell center near the wall with $y_{0}=1.9 \times 10^{-3}$ (indicated in Fig. 4). As in Fig. 2, the $x$ - and $z$-coordinates of the base location $\mathbf{x}_{0}$ are immaterial due to the homogeneity of the streamwise and spanwise directions. Figure 5a suggests that the two-point correlations obtained in case $\mathrm{F}$ (mesh spacing $0.5 \Delta y$ ) are similar to those of the baseline case. This observation is consistent with the findings from the other cases (A-E) with mesh refinement in the two homogeneous directions ( $x$ and $z$ ). It is noted that the $\rho_{v v}(y)$ profiles are not as smooth as those presented in Fig. 2. This is because only time-averaging was performed for the presented $\rho_{v v}(y)$ profiles; periodic averaging is not justified due to the lack of homogeneity in the wall normal direction. By measuring the distance over which the correlation drops below 0.3, one can see that the eddy size in the wall direction is $l_{y}=0.035$ for both the baseline case and case F. With the same procedure used to obtain data in Tables 2 and 3 as discussed in Section 3.1.1, we counted the number of cells $N_{y}$ over which the correlations $\rho_{v v}(y)$ drop below 0.3 , which serves as an indication of the resolution in the wall-normal direction. It was found that $N_{y}$ is 12.5 for the baseline case (mesh $\Delta y$ ) and 23 for case $\mathrm{F}$ (mesh $0.5 \Delta y$ ). Considering that case $\mathrm{F}$ has a mesh twice as fine as the baseline case, this finding also demonstrates that the eddy sizes implied by the two-point correlations in the two cases correspond very well. From Fig. 5b, the requirement of positive response of length-scale resolution can be verified, as the resolution indicator $\phi_{y}^{(l)}$ increases by a factor of two compared to the baseline case.

To highlight the inhomogeneous nature of the wall-normal direction, the two-point correlations $\rho_{\mathrm{vv}}(y)$ for two additional base points $y_{0}=0.055$ and $y_{0}=0.44$ are presented in Fig. 6. They are consistent with the studies for the $x$-and $z$-directions shown in Section 3.1.1. The offset $y$ is defined such that the points above the base point have positive $y$. For the case $y_{0}=0.055$ the two-point correlations are presented for offset values between $y=-0.055$ (corresponding to the bottom wall) and $y=0.945$ (channel center). Similarly, the offset range for $y_{0}=0.44$ is between -0.44 (bottom wall) and 0.56 (channel center). Two observations can be readily made from Fig. 6: (i) the distance over which $\rho_{\mathrm{vv}}$ drops below 0.3 is much larger in the $y_{0}=0.44$ case than in the case of $y_{0}=0.055$;

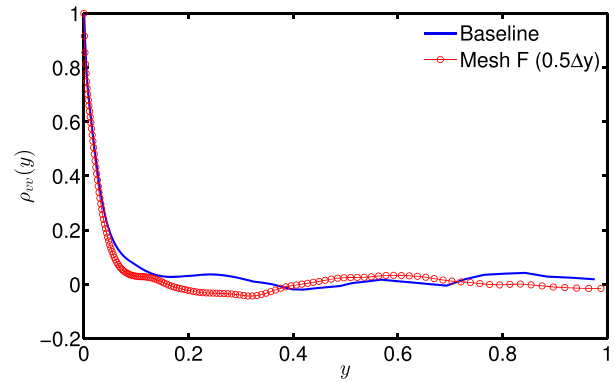

(a)

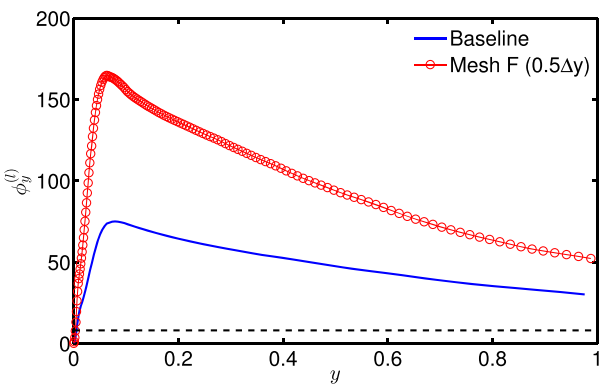

(b)

Fig. 5 Effects of wall-normal mesh refinement on mesh resolution indicators, showing the profiles of (a) the two-point correlations $\rho_{\mathrm{Vv}}(y)$ and (b) the resolution indicator $\phi_{y}^{(l)}$ along the wall-normal direction for the baseline case and case $\mathrm{F}$. The dashed line indicates threshold value 8 . The base location $\mathbf{x}_{0}$ in Eq. (12), is chosen as the first cell center next to the wall, as indicated in Fig. 4 , with $y_{0}=1.9 \times 10^{-3}$ 


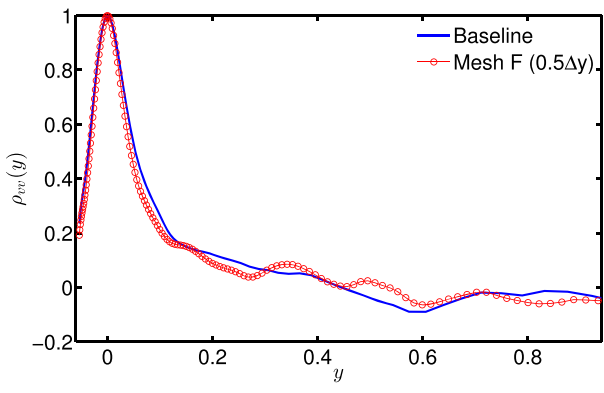

(a)

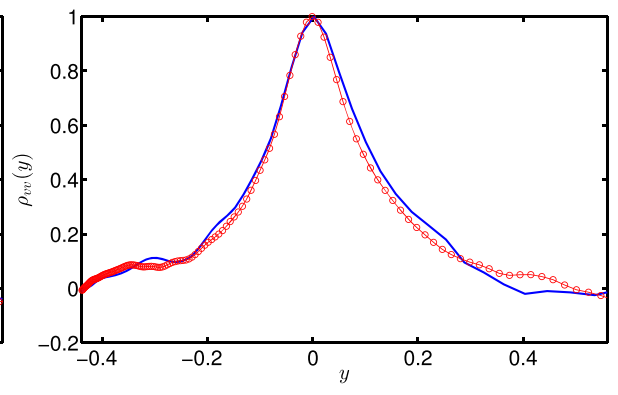

(b)

Fig. 6 Two point correlation $\rho_{\mathrm{vv}}(y)$ along the wall-normal direction for base point with (a) $y_{0}=0.055$ and (b) $y_{0}=0.44$. The offset $y$ is defined positive above the base point and negative below the base point

and (ii) mesh $\mathrm{F}$ and the baseline mesh show almost identical two-point correlation structures. Both observations are consistent with those in the homogeneous directions. A subtle yet notable difference is that the $\rho_{\mathrm{vv}}$ profiles are not exactly symmetric with respect to the base point, particularly when the offset distances are larger, although both curves are indeed rather close to being symmetric in the immediate vicinity of the base point.

From the analysis above we can conclude that the observed trends and findings based on the two homogeneous ( $x$ - and $z$-) directions still hold in the study of the inhomogeneous wall-normal direction.

\subsubsection{Consistency between the two resolution criteria}

Having examined the two resolution indicators individually, two questions follow naturally:

1. What does the two-point correlation in Fig. 2 and the derived "resolution indicator" presented in Tables 2 and 3 suggest about the resolution adequacy of each mesh?

2. Do they agree with each other on which regions are well-resolved and which are underresolved?

To answer the first question one needs to choose the threshold value $n_{0}$ for the number of cells required to resolve the eddy. Similar to the choice of $\phi_{0}$, also the choice of $n_{0}$ is subjective, but numbers between 3 and 12 seem to be reasonable. Due to the generally low resolution in this case, we use $n_{0}=6$ to facilitate the following discussion. However, most of the statements hold regardless of the threshold value chosen.

If we require $n_{0}=6$, from Table 2 it can be seen that the streamwise resolutions are inadequate for the baseline and for the meshes $\mathrm{B}$ and $\mathrm{D}$, while mesh $\mathrm{E}$ is marginally inadequate $\left(n=3.5\right.$ at $y_{0}=0.055, n=5$ at $\left.y_{0}=0.44\right)$. From Table 3 , we can similarly conclude that all meshes are well-resolved at $y_{0}=0.44$ while only meshes B, E, and D are well-resolved at $y_{0}=0.055$. Similar analysis can be made for other $y$ locations. However, we focus on the two representative locations for clarity. Assuming the wall-normal resolution is adequate for most of the domain outside the viscous sub-layer, one can conclude that mesh $\mathrm{C}$ has the best overall resolution. This conclusion would not change if another $n_{0}$ is used, and it is consistent with our previous analysis in Section 3.1.2 based on resolution indicator $\phi_{\min }^{(l)}$. In addition, the following observations can be made: (1) meshes with $x$ refinement (A and C) are under-resolved at $y_{0}=0.055$ but well-resolved at $y_{0}=0.44$ (although marginally since $n=5$ for mesh E); (2) meshes with $z$ refinement (B and D) as well as the baseline mesh 
are under-resolved at both $y$ locations. From Fig. 3d, one could reach these two conclusions only when a larger threshold value (e.g., $\left.\phi_{0}=12\right)$ was taken.

This seems to suggest that the resolution indicator $n$ implied by two-point correlation is smaller than $\phi_{\min }^{(l)}$. In other words, "eddy sizes" obtained from two-point correlation are smaller than that estimated from $k$ and $\varepsilon$. Consequently, $\phi_{0}$ should be larger than $n_{0}$ for the two criteria to reach consistent conclusions. Overall, however, the two criteria do show some qualitative agreement, and thus the resolution indicator based on turbulent length-scale $\phi^{(l)}$ is a promising criterion for hybrid simulations, although there are still some open questions that need to be answered. Pope [11] (Chapter 6.5.7) pointed out that the ratio of longitudinal integral length scale $L_{11}$ to $l_{t}=k^{3 / 2} / \varepsilon$ depends on Reynolds numbers up to a upper limit $R e_{\text {lim }} \approx 5000$, with $L_{11}$ being smaller throughout the entire Re range. While an anisotropic version of $l_{t}$ is used in this study, and thus the exact ratio here would be different, we note (1) that our observation above qualitatively agrees with that of Pope [11]; and (2) that the Reynolds number based on bulk velocity and half channel width in this case (approximately $2 \times 10^{5}$ ) is higher than the limit Reynolds number $R e_{\text {lim }}$.

\subsection{Periodic hill flow at $R e=10595$}

A representative case of complex flows with massive separation is the flow over periodic hills, which is chosen as a benchmark case by a French-German research group on LargeEddy Simulation of Complex Flows [20].

The geometry of the computational domain is shown in Fig. 7 and the detailed description of the hill shape can be found in ref. [21]. Resolutions of the five meshes investigated in this case are presented in Table 4. These cases have the following resolution in wall-normal (y) direction: $N_{y}=90, \Delta y_{\min }^{+}=1$, and $\Delta y_{\max }^{+}=7.3$. All length scales and coordinates are normalized by the crest height $H$ of the hill.

Different from the plane channel flow case, this flow is statistically homogeneous only in the spanwise $(z)$ direction. The two-point correlation curves depend on the streamwise and the wall-normal coordinates $x_{0}$ and $y_{0}$ of the base location, while in contrast they only depend on $y_{0}$ in plane channel flows. This additional dimension of inhomogeneity makes it more difficult to infer resolution of each cell based on the correlation curves.

As an illustration, we present the two-point correlations at $y_{0}=0.125$ and $y_{0}=1$ for the five meshes in Fig. 8, with basis location $x_{0}=2$. The quantitative feature of the correlation

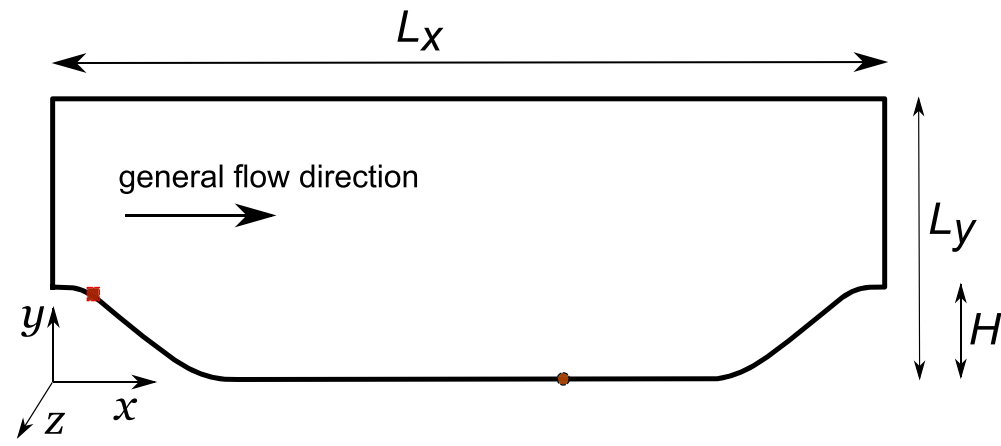

Fig. 7 Schematic of flow over periodic hills test case. The square and the circle indicate the approximate locations of flow separation and reattachment, respectively. The dimensions of the domain are: $L_{x}=9$, $L_{y}=3.036$, and $L_{z}=4.5$ (all normalized by the crest height $H$ of the hill) 
Table 4 Number of cells in streamwise and spanwise directions and the resolutions in viscous units for flow over periodic hills case

\begin{tabular}{lllll}
\hline mesh & $\mathrm{N}_{\mathrm{X}}$ & $\mathrm{N}_{\mathrm{z}}$ & $\Delta x^{+}$ & $\Delta z^{+}$ \\
\hline baseline & 74 & 36 & 60.8 & 62.5 \\
$0.5 \Delta x(\mathrm{~A})$ & 148 & 36 & 30.4 & 62.5 \\
$0.5 \Delta z(\mathrm{~B})$ & 74 & 72 & 60.8 & 31.3 \\
$0.25 \Delta x(\mathrm{C})$ & 296 & 36 & 15.2 & 62.5 \\
$0.25 \Delta z$ (D) & 74 & 144 & 60.8 & 15.6 \\
\hline
\end{tabular}

curves are the same as those in the plane channel case. However, while the conclusion about the resolution adequacy based on a two-point correlation curve at a particular $y$ coordinate should be valid for all cells in the plane at this wall-normal location, the conclusions for this case would be only valid for the cells along the line with a particular $x$ and $y$ coordinate. Due to this limitation, we do not present further analysis of the two-point correlations for this case.

On the other hand, the resolution indicator $\phi^{(l)}$ can still be examined against the requirements outlined in Section 1. To this end, $\phi^{(l)}$ was computed at each time step, and temporal and spanwise averaging were performed to obtain the mean values. The indicator $\phi_{z}^{(l)}$ is presented as contour plot in Fig. 9 for the baseline mesh and the meshes B $(0.5 \Delta z)$ and D $(0.25 \Delta z)$. Two contour lines of $\phi_{z}^{(l)}=6$ and $\phi_{z}^{(l)}=8$ are also shown. The dark regions encircled by the contour lines would be the under-resolved region (RANS region) in hybrid simulations if a threshold corresponding to the value of the contour line was chosen for $\phi_{z}^{(l)}$. It can be seen that the under-resolved region, if defined based on $\phi_{z}^{(l)}$, shrinks in response to the refinements in $z$ direction, similar to what has been observed in Fig. 3 for plane channel flows. This is the desired response of a good resolution indicator under the positive response requirement. Similar contours for the streamwise direction are presented in Fig. 10 for meshes A $(0.5 \Delta x)$ and $\mathrm{C}(0.25 \Delta x)$, showing the same trend. They serve as confirmation for the behavior of components of $\phi^{(l)}$ observed in the plane channel flow case.

\subsection{Influence of model constants}

Another important criterion for resolution indicators is the sensitivity on model parameters. In most eddy-viscosity SGS models, the turbulent quantities are directly (e.g., for $k$ and $\varepsilon$ )

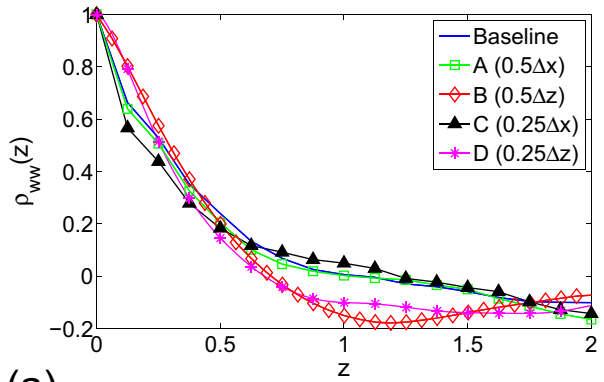

(a)

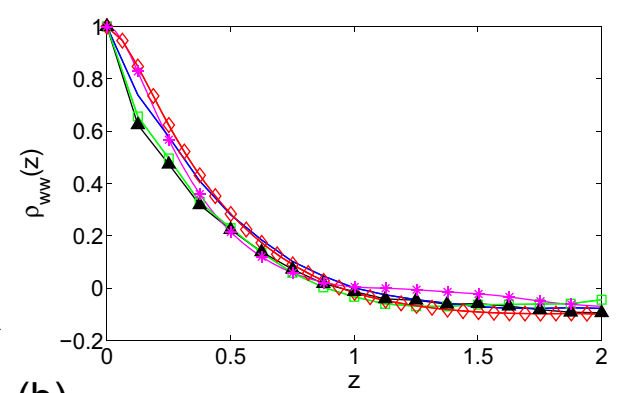

(b)

Fig. 8 Normalized spanwise two-point correlation $\rho_{\mathrm{ww}}(\mathrm{z})$ for periodic hill flow of $R e=10595$. The results are presented for two wall-normal base point locations: (a) $y_{0}=0.125\left(y^{+}=63\right)$ and (b) $y_{0}=1\left(y^{+}=500\right)$. The streamwise coordinate of the base point is $x_{0}=4.5$ 


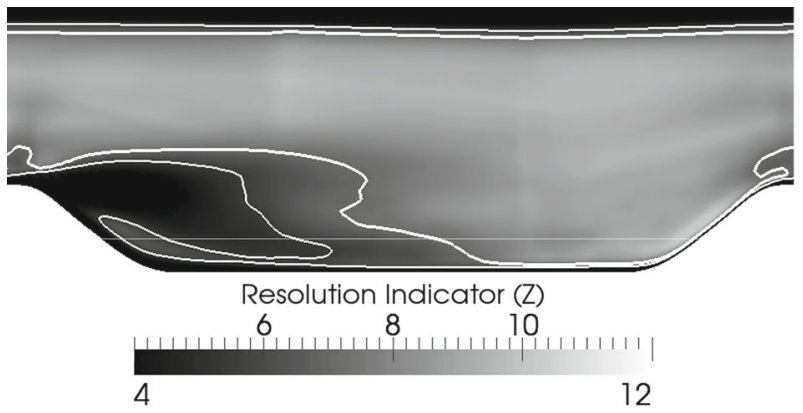

(a) Baseline

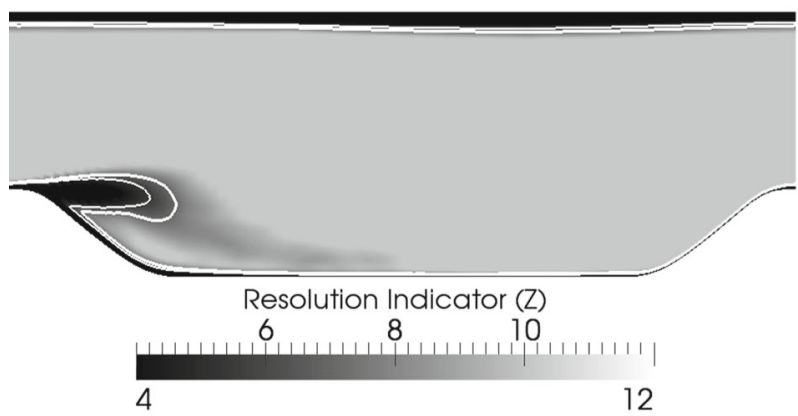

(b) Mesh B $(0.5 \Delta z)$

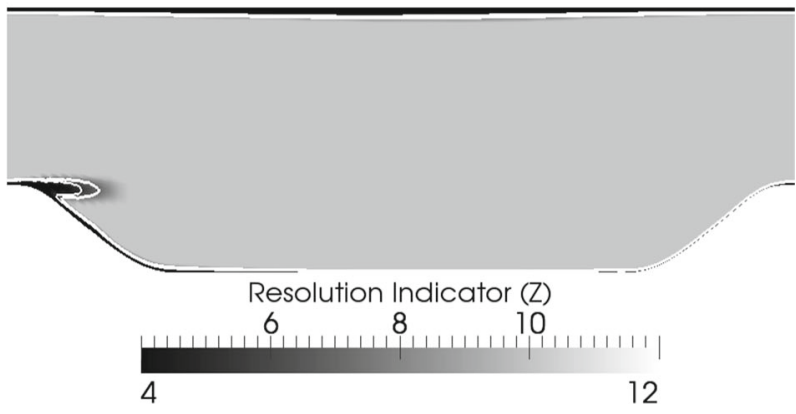

(c) Mesh D $(0.25 \Delta z)$

Fig. 9 Contour plots for resolution indicator $\phi_{z}^{(l)}$ with contour lines for $\phi_{z}^{(l)}=6$ (thin lines) and $\phi_{z}^{(l)}=8$ (thick lines)

or indirectly (e.g., for $l_{t}$ ) influenced by the model constants (e.g. $C_{k}$ and $C_{\varepsilon}$ in OEE model; $C_{s}$ in Smagorinsky model). This is true for both static and dynamic models, although the effects in the latter may be subtle. In this subsection, we investigate the influences of model constants $C_{k}$ on the resolution indicators in a static OEE model.

To study the effects of model constants on resolution indicators, simulations of the plane channel flow (See Section 4.1) were conducted with two typical values: $C_{k}=0.094$ (the default) and $C_{k}=0.05$ (corresponding to $C_{s}=0.1$ in Smagorinsky model; the reduced value is often used for wall-bounded flows). The simulations with both $C_{k}$ values were conducted on two meshes: the baseline mesh and mesh B $(0.5 \Delta z)$. To place the influence of 


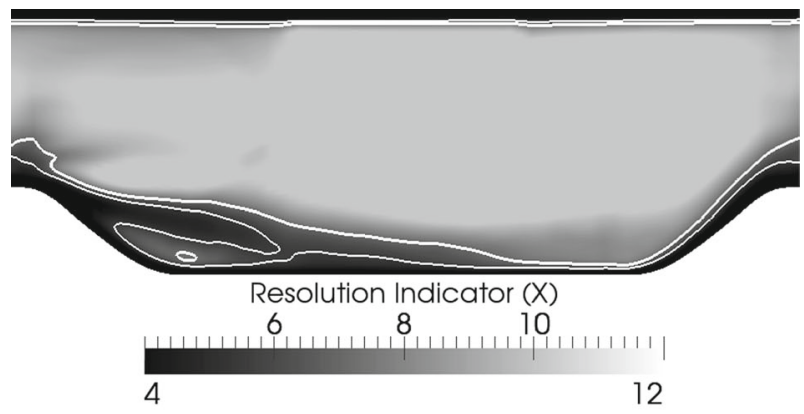

(a) Baseline

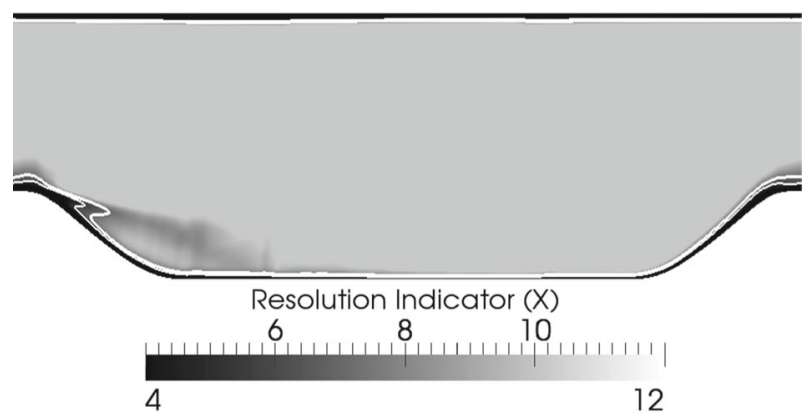

(b) Mesh A $(0.5 \Delta x)$

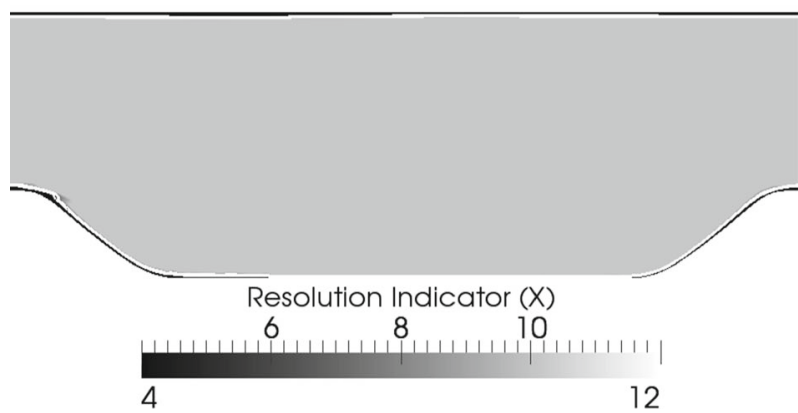

(c) Mesh C $(0.25 \Delta x)$

Fig. 10 Contour plot for resolution indicator $\phi_{x}^{(l)}$ with contour lines for $\phi_{x}^{(l)}=6$ (thin lines) and $\phi_{x}^{(l)}=8$ (thick lines)

$C_{k}$ in context, the difference in $\phi$ caused by using different $C_{k}$ are compared to those caused by refinement in $z$ direction. The latter can be loosely interpreted as the normalization basis.

The mean profiles are presented in Fig. 11 for resolution indicators $\phi_{k}$ and $\phi_{\varepsilon}$ based on percentages of resolved turbulent kinetic energy $k$ and dissipation $\varepsilon$ as

$$
\begin{aligned}
\phi_{k} & =\frac{k^{\mathrm{res}}}{k^{\mathrm{res}}+k^{\mathrm{sgs}}} \text { and } \\
\phi_{\varepsilon} & =\frac{\varepsilon^{\mathrm{res}}}{\varepsilon^{\mathrm{res}}+\varepsilon^{\mathrm{sgs}}},
\end{aligned}
$$




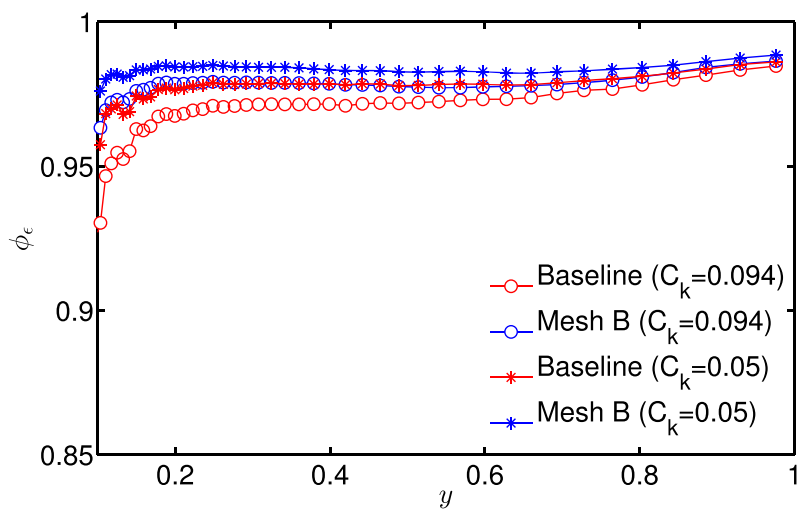

(a)

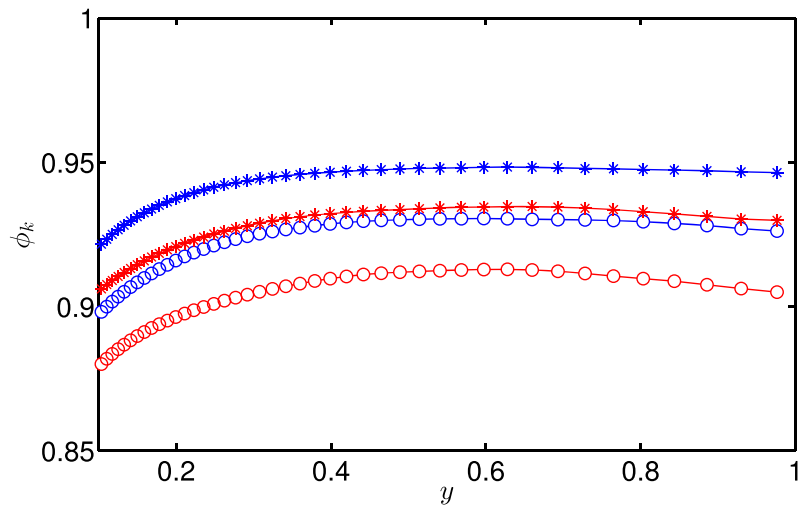

(b)

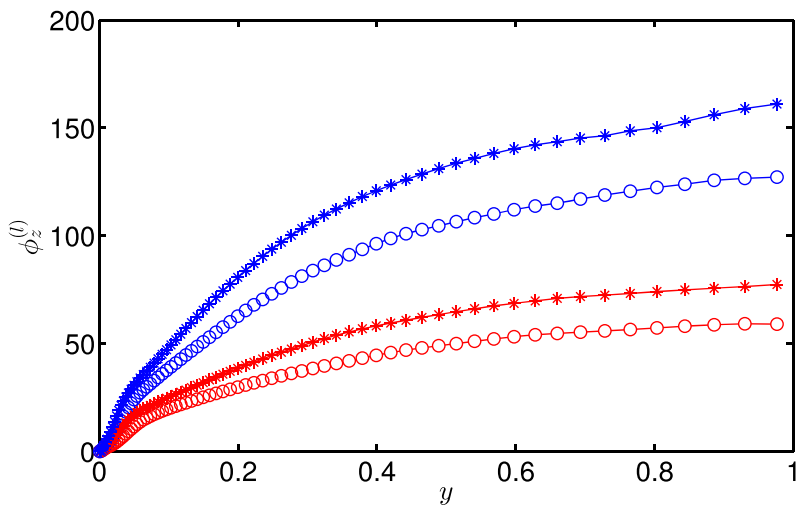

(c)

Fig. 11 Influence of model coefficient $C_{\mathrm{k}}$ on resolution indicators $\phi_{\varepsilon}$ (plot a), $\phi_{k}$ (plot b), and $\phi_{z}^{(l)}$ (plot c) for the plane channel flow of $R e_{\tau}=4000$

respectively, and for $\phi^{(l)}$ as defined in Eq. (10). The superscripts 'res' and 'sgs' indicate resolved and SGS components, respectively. 
The influence of $C_{k}$ on the resolution indicators are represented by the gap between the lines of the same color (or grey scale) but different symbols. The influences of mesh refinement are represented by the gap between lines of the same symbol but different colors (or grey scales). It can be seen that the influence of mesh refinement and that of varying $C_{k}$ are comparable for $\phi_{k}$ and $\phi_{\varepsilon}$. For example, for both indicators, if we regard the baseline mesh with $C_{k}=0.094$ as the base case, decreasing $C_{k}$ to 0.05 leads to the same increase of resolution indicator as refining the mesh. On the other hand, for $\phi_{z}^{(l)}$ as shown in Fig. 11c, the influence of $C_{k}$ is much smaller than that of the mesh refinement.

To confirm the observations above, additional simulations were conducted with mesh $\mathrm{D}(0.25 \Delta z)$ as well, and results for $\phi_{x}^{(l)}$ were analyzed similarly. These additional results show the same trend as discussed above, and thus are omitted for the sake of brevity.

The reason for the stronger dependence of $\phi_{k}$ and $\phi_{\varepsilon}$ on $C_{k}$ is that they are defined based on $k^{\mathrm{sgs}}$ and $\varepsilon^{\mathrm{sgs}}$, which are modeled as directly proportional to $C_{k}^{3 / 2}$. Another factor is that the definitions in Eqs. (14) and (15) neglect the numerical components of $k$ and $\varepsilon$, which are difficult to quantify. This effect is discussed in more details in the literature [4, 5, 22]. In contrast, $\phi^{(l)}$ is defined based on total turbulent kinetic energy and total dissipation. The direct influence of $C_{k}$ is limited to the SGS part, which is relatively small.

Based on the observations it is concluded that compared to $\phi_{k}$ and $\phi_{\varepsilon}$ the resolution indicator $\phi^{(l)}$ is slightly less susceptible to the variations of model constant $C_{k}$, and is thus the preferred choice in this aspect.

\section{Resolution Indicators in Hybrid Simulations}

As explained in Section 1, an intrinsic difficulty of dynamic resolution evaluation is due to the possible interactions between the resolution indicators and the solutions. The extent of interactions would naturally depend on the role of resolution indicators in the hybrid solvers and on the coupling between LES and RANS components within the solver, and thus no general conclusion can be made. In this section we study these potential interactions in a dual-mesh consistent hybrid LES/RANS framework presented in ref. [2]. Figure 12 is used to illustrate the basic ideas of the hybrid framework. In this framework, LES and RANS simulations are conducted simultaneously on the same domain with different meshes. This feature is indicated by the two time axes (for LES and RANS, respectively) in Fig. 12. The LES mesh is designed to adequately resolve the free-shear regions but not the near-wall region; the RANS mesh is designed to resolve the near-wall region but not the free-shear region. The cells are classified as well-resolved or under-resolved cells (or LES and RANS regions, indicated in Fig. 12 by wavy and checkerboard patterns, respectively) based on the resolution indicators computed from LES data dynamically. In the under-resolved cells (i.e., the RANS region), drift terms are applied to relax the averaged LES solution towards the RANS solutions (first moment, i.e., velocity, and second moment, i.e., Reynolds stresses or turbulent kinetic energy). Similarly, in the well-resolved region the RANS solutions are forced towards the average of LES solutions. The averaged LES quantities are obtained by averaging LES data after each time-step, which are then used together with RANS quantities to compute relaxation forces. This selective dominance mechanism is also illustrated in Fig. 12.

A brief formulation of the hybrid framework including the equations and the expressions of the forcing is presented below. For more details the reader is referred to 


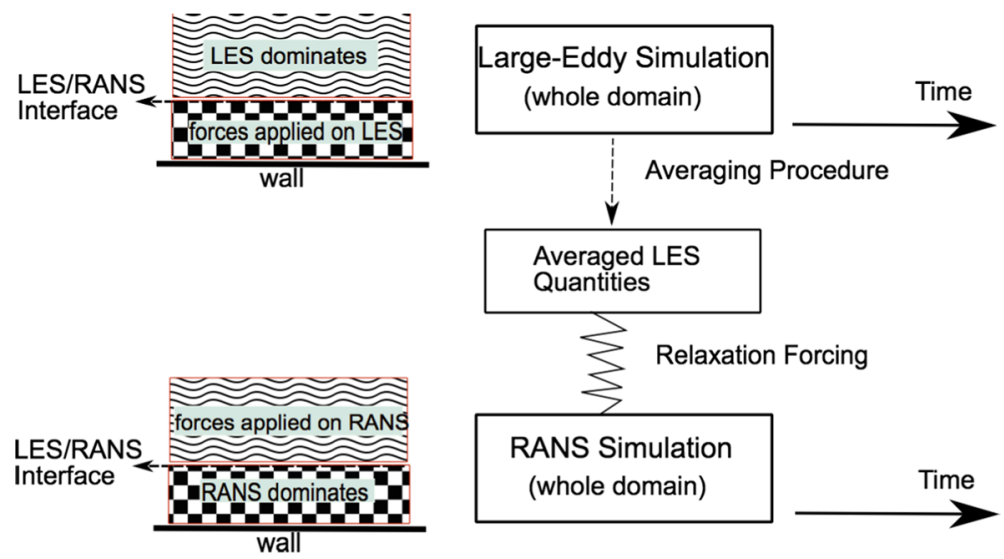

Fig. 12 A diagram of the hybrid LES/RANS framework. The two time axes indicate that LES and RANS (denoted by the top and bottom boxes) are conducted simultaneously on the entire domain. The middle box (averaged LES quantities) indicates quantities obtained by post-processing (i.e., averaging) LES data after each time-step. The averaged LES quantities are then used together with RANS quantities to compute relaxation forces. The drawings on the left illustrate the "selective dominance" mechanism on in LES and RANS simulations

ref. [2]. In this study, the performance of the proposed resolution indicator is thoroughly investigated.

\subsection{Summary of consistent hybrid les/rans framework}

For incompressible flows with constant density, the momentum and pressure equations for the filtered and the Reynolds-averaged quantities can be written in an unified form as follows:

$$
\begin{gathered}
\frac{\partial U_{i}^{*}}{\partial t}+\frac{\partial\left(U_{i}^{*} U_{j}^{*}\right)}{\partial x_{j}}=-\frac{1}{\rho_{f}} \frac{\partial p^{*}}{\partial x_{i}}+v \frac{\partial^{2} U_{i}^{*}}{\partial x_{j} \partial x_{j}}-\frac{\partial \tau_{i j}^{*}}{\partial x_{j}}+Q_{i}^{*} \\
\text { and } \frac{1}{\rho_{f}} \frac{\partial^{2} p^{*}}{\partial x_{i} \partial x_{i}}=-\frac{\partial^{2}}{\partial x_{i} \partial x_{j}}\left(U_{i}^{*} U_{j}^{*}+\tau_{i j}^{*}\right)+\frac{\partial Q_{i}^{*}}{\partial x_{i}},
\end{gathered}
$$

where $t$ and $x_{i}$ are time and space coordinates, respectively; $v$ is the kinematic viscosity, $p^{*}$ is the pressure, and $\rho_{f}$ is the constant fluid density. In the filtered equations, $U_{i}^{*}, p^{*}$, and $\tau_{i j}^{*}$ represent filtered velocity $\bar{U}_{i}$, filtered pressure $\bar{p}$, and residual stresses $\tau_{i j}^{\text {sgs }}$ plus filtered viscous stresses $\bar{\tau}_{i j}$, respectively. In the Reynolds averaged equations, $U_{i}^{*}, p^{*}$, and $\tau_{i j}^{*}$ represent Reynolds-averaged velocity $U_{i}^{\text {RANS }}$, Reynolds-averaged pressure $p i^{\text {RANS }}$, and Reynolds stress $u_{i}, u_{j}{ }^{\text {RANS }}$ plus mean viscous stress, respectively. $Q_{i}^{*}$ represents the drift terms applied in the filtered equations $\left(Q_{i}^{L}\right)$ and in the Reynolds averaged equations $\left(Q_{i}^{R}\right)$ to ensure consistency between the two solutions. This term will be detailed in Eqs. (20) and (21). In this hybrid framework, filtered and Reynolds averaged equations are solved simultaneously in the entire domain but on separate meshes. The consistency between the two solutions is enforced via relaxation forcing in the respective equations. We first define 
exponentially weighted average quantities including velocity, dissipation, and turbulent stresses for the LES as

$$
\begin{aligned}
\left\langle\bar{U}_{i}\right\rangle^{\mathrm{AVG}}(t)= & \frac{1}{T} \int_{-\infty}^{t} \bar{U}_{i}\left(t^{\prime}\right) e^{-\left(t-t^{\prime}\right) / T} d t^{\prime}, \\
\langle\tau\rangle_{i j}^{\mathrm{AVG}}(t)= & \frac{1}{T} \int_{-\infty}^{t}\left[u_{i}^{\prime \prime}\left(t^{\prime}\right) u_{j}^{\prime \prime}\left(t^{\prime}\right)+\tau_{i j}^{\mathrm{sgs}}\left(t^{\prime}\right)\right] e^{-\left(t-t^{\prime}\right) / T} d t^{\prime}, \quad \text { and } \\
\langle\varepsilon\rangle^{\mathrm{AVG}}(t)= & \frac{1}{T} \int_{-\infty}^{t}\left[2 \nu \bar{S}_{i j}\left(t^{\prime}\right) \bar{S}_{i j}\left(t^{\prime}\right)-\tau_{i j}^{\mathrm{sgs}}\left(t^{\prime}\right) \bar{S}_{i j}\left(t^{\prime}\right)\right] e^{-\left(t-t^{\prime}\right) / T} d t^{\prime} \\
& -2 v\left\langle\bar{S}_{i j}\right\rangle^{\mathrm{AVG}}\left\langle\bar{S}_{i j}\right\rangle^{\mathrm{AVG}},
\end{aligned}
$$

respectively, where

$$
u_{i}^{\prime \prime}=\bar{U}_{i}-\left\langle U_{i}\right\rangle^{\mathrm{AVG}}
$$

is the fluctuating velocity with respect to the exponentially weighted average;

$$
\bar{S}_{i j}=\frac{1}{2}\left(\frac{\partial \bar{U}_{i}}{\partial x_{j}}+\frac{\partial \bar{U}_{j}}{\partial x_{i}}\right)
$$

is the resolved rate-of-strain tensor; and $\tau_{i j}^{\mathrm{sgs}}$ is the SGS stress tensor. This definition is consistent with those in Section 2.2, with the averaging operator specifically defined as exponentially weighted averaging. The terms in brackets inside the integral in Eq. (17b) are total turbulent stresses in LES. The consistency between the two solutions requires that the exponentially weighted average quantities and the Reynolds averaged quantities should be approximately equal, e.g., $\left\langle U_{i}\right\rangle^{\mathrm{AVG}} \approx\left\langle U_{i}\right\rangle$ for the velocities. The regions well-resolved by the LES mesh are classified as LES regions, where the LES solution would dominate, and the under-resolved regions are called RANS regions, where the RANS solution should prevail. The consistency and the selective dominance mechanisms are enforced via drift forces ( $Q_{i}^{L}$ in the filtered equations and $Q_{i}^{R}$ in Reynolds averaged equations) defined as follows:

$$
Q_{i}^{L}= \begin{cases}\left(\left\langle U_{i}\right\rangle^{\mathrm{RANS}}-\left\langle U_{i}\right\rangle^{\mathrm{AVG}}\right) / T^{(L)}+G_{i j}\left(\left\langle U_{j}\right\rangle^{\mathrm{AVG}}-\bar{U}_{j}\right) / T^{(G)} & \text { in RANS regions } \\ 0 & \text { in LES regions }\end{cases}
$$

and

$$
Q_{i}^{R}= \begin{cases}\left(\left\langle U_{i}\right\rangle^{\mathrm{AVG}}-\left\langle U_{i}\right\rangle^{\mathrm{RANS}}\right) / T^{(R)} & \text { in LES regions } \\ 0 & \text { in RANS regions }\end{cases}
$$

where

$$
G_{i j}=\frac{\left\langle\tau_{i j}\right\rangle^{\mathrm{AVG}}-\left\langle u_{i} u_{j}\right\rangle^{\mathrm{RANS}}}{\left\langle\tau_{k k}\right\rangle^{\mathrm{AVG}}},
$$

and $T^{(\mathrm{L})}, T^{(\mathrm{G})}$ and $T^{(\mathrm{R})}$ are the relaxation time scales. Drift terms are also added in a similar way to the equations for turbulent quantities such as $\tau_{i j}, k$ and $\varepsilon$ depending on the specific models. The motivation of the hybrid framework, interpretations of the forcing terms, and the detailed solution algorithms are presented in ref. [2].

The framework is general and can conveniently accommodate any SGS and RANS turbulence models. In the hybrid simulations presented in Section 4, the same one-equation-eddy model as in the pure LES presented in Section 3 was adopted. For the RANS solver, the Reynolds stress model with elliptic relaxation by Durbin [24] was used. Averaging and relaxation time scales were $T=2.2, T^{(L)}=T^{(R)}=0.28$ and $T^{(G)}=0.07$; all normalized by $H / U_{b}$. This choice of parameters followed ref. [2], where the relative insensitivity of results to parametric perturbations has been demonstrated. 
Although the merits and shortcomings of the dual-mesh hybrid LES/RANS framework are not the main focus of this work, a few remarks are noted here. Compared with pure LES and other single-mesh hybrid methods, the dual-mesh hybrid solver introduced here needs to compute the drift terms and to solve the RANS equations additionally, albeit on a much coarser grid. The overhead due to these operations is $30 \%$ of the total computational cost for the simulations conducted in ref. [2]. For high Reynolds number flows, however, the relative percentage of the overhead is expected to diminish. For more details the readers are referred to ref. [2].

\subsection{Performance of resolution indicator in the hybrid simulation}

Ideally we expect that the relaxation forcing applied to LES in the under-resolved region does not significantly alter the turbulence statistics in the well-resolved region. To assess the influence of the coupling on the turbulent structures in the well-resolved region, simulations were conducted using the dual-mesh hybrid LES/RANS solver developed according to the framework above. The resolution for the LES and the RANS meshes are specified in Table 5. The simulations were conducted with a time span of four flow-through times, and the temporal and spanwise averaged quantities are presented here. For the convenience of comparison with previous results, the same LES mesh as in ref. [2] is used. Since the resolution adapted here is very low in the entire domain, a small threshold value $\phi_{0}=2$ was used to avoid an excessively large RANS region. This is also to reduce the size of the region behind the separation point to be classified as RANS cells. In addition, results were improved by refining the mesh in spanwise $z$ direction. It is found that scaling $\phi_{z}$ by a factor of three (essentially using a threshold value of $\phi_{0} / 3$ for $\phi_{z}$ ) gave the same results as using the refined mesh, suggesting that in this criterion a smaller threshold value for $z$ direction may be used. Here, to save computational cost $\phi_{z}$ was scaled instead of refining the mesh. Admittedly, this setting is not rigorous, and according to the studies in Section 3 we recommended threshold values ranging from 3 to 12 in hybrid LES/RANS simulations.

The two-point correlations are presented for two wall-normal locations $y_{0}=1$ and $y_{0}=2$ in Fig. 13. Both are located in the free shear region. The streamwise correlation $\rho_{u u}(x)$ and spanwise correlations $\rho_{w w}(z)$ are presented in plots (a) and (b), respectively. Note that the streamwise $(x)$ direction is not statistically homogeneous, and the results were obtained at the base streamwise location of $x_{0}=2$. For both $\rho_{u u}(x)$ and $\rho_{w w}(z)$, as shown in Fig. 13a and b, no major differences are observed between the pure LES results and the hybrid results, particularly in the region where significant correlation exists. This suggests that the forcing in the RANS region does not change the turbulent structures in the LES region significantly.

In Figs. 14 and 15, we further compare the resolution indicators $\phi^{(l)}$ obtained from pure LES and hybrid simulations using the same mesh and setup. The streamwise and spanwise components $\left(\phi_{x}^{(l)}\right.$ and $\left.\phi_{z}^{(l)}\right)$ are shown in grey scale contours. Two contour lines corresponding to two values for the components of $\phi^{(l)}$ are also shown. The dark

Table 5 The resolution of the LES and RANS meshes in the hybrid simulation of flow over periodic hills

\begin{tabular}{llll}
\hline & $N_{x}$ & $N_{y}$ & $N_{z}$ \\
\hline LES & 74 & 37 & 36 \\
RANS & 128 & 37 & 18 \\
\hline
\end{tabular}




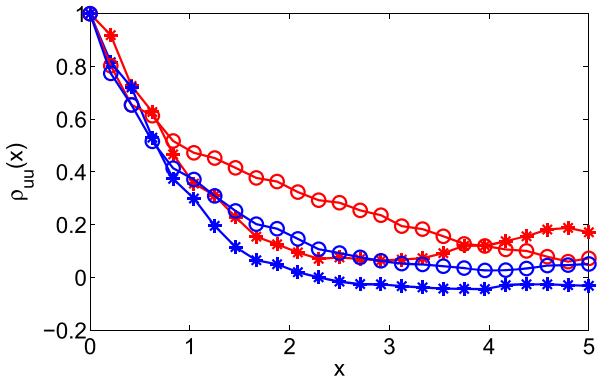

(a)

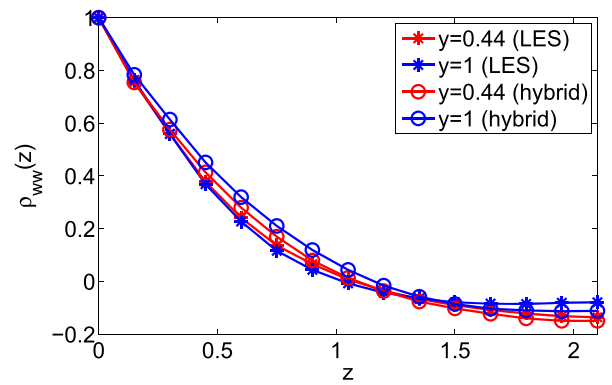

(b)

Fig. 13 Influence of the LES/RANS coupling on the turbulence structures represented by the differences between the two-point correlations in pure LES and those in hybrid LES/RANS simulations. The sampling points are all located in the well-resolved (LES) region where no relaxation forcing is applied

regions encircled by the contour lines (or between the contour lines and the wall boundaries) indicate under-resolved (RANS) region in simulations, if threshold values corresponding to the contour lines were used. The results suggest that the RANS regions indicated by $\phi_{x}^{(l)}$ and $\phi_{z}^{(l)}$ are similar in pure LES and hybrid simulations except for the region immediately after the separation (e.g., the dark pocket for $\phi_{z}^{(l)}$ in Fig. 15). This region is characterized by strong instability and the breakdown of separated boundary layer, and is challenging for any resolution evaluations [10].

In Fig. 16, the mean velocity profiles obtained using the hybrid solver are presented at eight cross sections. The benchmark results obtained using highly resolved LES [23] and those obtained using LES on the same mesh are also shown for comparison. It can be seen that the improvement over pure LES is clear, particularly near the upper wall and at the center of the channel. This observation is similar to the results presented in ref. [2] where a static criterion solely based on wall-distance was used. However, the prediction near the reattachment point $(x=6)$ is not as satisfactory as using the wall-distance criterion. This is also related to the region after the separation since the eddies impinging the bottom wall here come from the vortex breakdown resulting from the separated boundary layer. If that region was classified as RANS region by the resolution indicator, the prediction quality would deteriorate. Better resolution evaluation is needed in this region, ideally without resort to wall distance for reasons explained before.

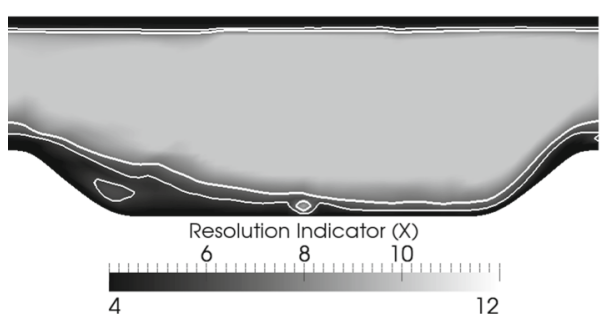

(a)

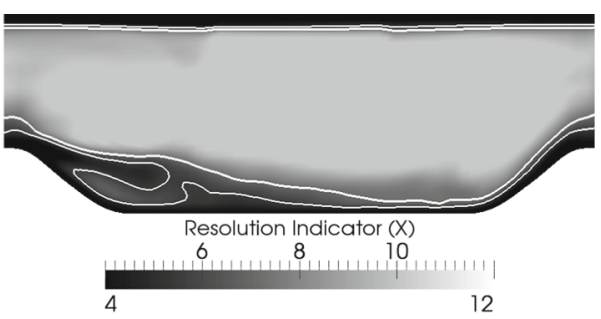

(b)

Fig. 14 Comparison of resolution indicators $\phi_{x}^{(l)}$ in pure LES and hybrid LES/RANS simulations on the same mesh, demonstrating the interactions between resolution indicators and hybrid solvers 


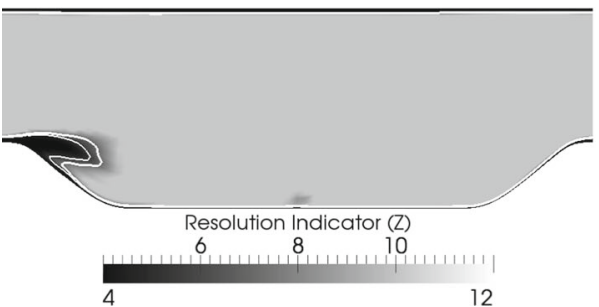

(a)

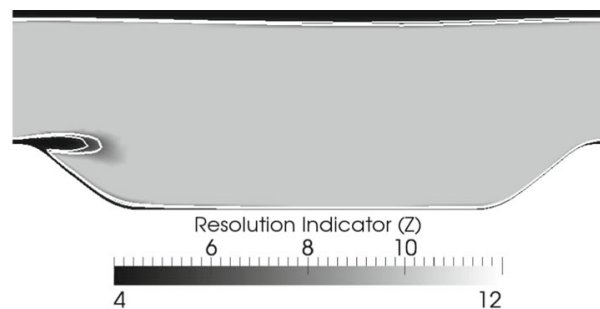

(b)

Fig. 15 Comparison of resolution indicators $\phi_{z}^{(l)}$ in pure LES and hybrid LES/RANS simulations on the same mesh, demonstrating the interactions between resolution indicators and hybrid solvers

Overall, the similarity between the two-point correlations and resolution indicators obtained from pure LES and those from hybrid simulations suggests that the interactions between the resolution evaluation and the solutions are moderate. The favorable comparison of the velocity profiles obtained using dynamic resolution evaluation with the benchmark solution also demonstrated the potential of this criterion. These observations lend preliminary support to the use of dynamic resolution evaluation in hybrid LES/RANS simulations and to the merits of the modified length-scale based criterion. However, the studies here are limited to one specific hybrid solver and thus caution must be exercised when generalizing these findings to other hybrid frameworks.

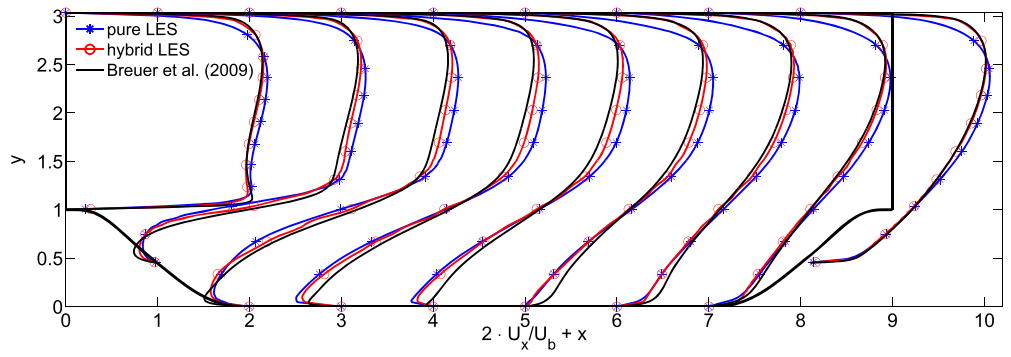

(a)

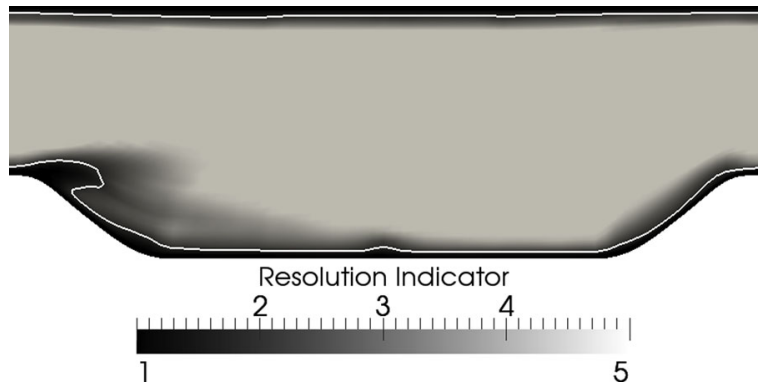

(b)

Fig. 16 a Mean velocity profiles at eight cross sections ( $x=0-8$ with intervals of length 1$)$ obtained using the consistent hybrid LES/RANS solver compared to pure LES on the same mesh and benchmark results of Breuer et al. [23]. b Contour plot for resolution indicator $\phi_{\min }^{(l)}$ with the contour line of $\phi_{\min }^{(l)}=2$ indicating the mean location of the LES/RANS interface during the simulation 


\section{Discussion}

In this section, we discuss several important issues related to the proposed resolution evaluation method, its robustness and practical implementation in hybrid solvers.

\subsection{Near-wall modeling}

It is noted that the resolution evaluation methods are developed for LES (usually with inadequate near-wall resolutions) in the context of hybrid LES/RANS simulations. Since in hybrid simulations RANS essentially serves as wall model for the LES, it is reasonable to assume that the LES are conducted without applying empirical wall models such as the Wall-Adapting Local Eddy-viscosity (WALE) model [25]. In wall-modeled LES, the turbulent stresses and/or velocities in the near-wall cells are specified or modified according to empirical models, which may mislead the resolution evaluation procedure since resolution indicators depend on the stresses and velocities. This limitation equally applies to other resolution evaluation methods if they are used to assess cell resolutions $[8,9]$.

\subsection{Compromise between mathematical rigorousness and convenience}

In this study, we used only the diagonal terms of $L_{i j}$ (see Eq. (5)) to characterize the turbulent eddy size. However, $L_{i j}$ is not diagonally dominant for all turbulent flows, and thus the this compromise is not mathematically rigorous. Take the plane channel flow at $R e=13750$ $\left(R e_{\tau}=395\right)$ for example, according to Pope [11] (Chapter 7.1.6) and Kim et al. [26], in the viscous wall region (approximately between $y^{+}=0$ and 50) the shear component $\left\langle u_{i} u_{j}\right\rangle$ of the Reynolds stress is larger than the wall normal component. However, in most part of the flow field away from the wall, the Reynolds stress tensor is indeed diagonally dominant. On the other hand, the anisotropy of the three normal components of $\left\langle u_{i} u_{j}\right\rangle$ is evident $[11,26]$, which corresponds well with the physical anisotropy of the eddies. Therefore, by making a compromise between mathematical rigorousness and practical convenience of implementation, using the anisotropic component-wise criterion is expected to provide a more physical description of eddy structures than the isotropic formulation does.

\subsection{Implementation in hybrid les/rans solver}

When evaluating resolution in hybrid simulations, it is important to avoid two unphysical phenomena:

1. "blinking cells", i.e. the classifications of some cells switch between LES (wellresolved) cells and RANS (under-resolved) frequently; and

2. "isolated cells", i.e., one or a few RANS (or LES) cells are surrounded by LES (or RANS) cells.

A temporally steady and spatially clearly defined LES/RANS interface should be obtained instead. When blinking or isolated cells appear, the turbulent kinetic energy transfer between resolved and modeled scales would not occur correctly. However, although the formulation of the resolution evaluation method proposed in this study does not guarantee that the cell classification results will be free from the two phenomena, according to our experiences they generally do not cause major issues in our simulations. This is because the resolution indicators are defined based on averaged LES quantities (an exemplary averaging procedure is defined in Eq. (17)), and not on the instantaneous filtered quantities. The averaging 
procedure eliminates high-frequency signals (which may cause blinking), as well as small wave-number features (which may lead to isolated cells). Moreover, to avoid blinking for the cells with resolution indicators close to the specified threshold $\phi_{0}$, a small "friction" was applied when switching a cell between LES and RANS. Specifically, a RANS cell is only switched to become an LES cell if the resolution indicator value is larger than $(1+\gamma) \phi_{0}$, where $\gamma$ is a number much smaller than 1 (in this study $\gamma=0.05$ was used); similarly, an LES cell is only switched to become a RANS cell if its resolution indicator $\phi$ is smaller than $(1-\gamma) \phi_{0}$. This hysteresis effect is desirable to avoid blinking, but it also raises the question of initialization: how the cells should be classified at $t=0$.

To answer this question, one needs to bear in mind that the averaging procedure in Eq. (17) used in this work also has the initialization issue, which limits the application of the consistent hybrid solver mostly to statistically stationary flows. For flows with coherent structures, the periods of the coherent eddies have to be much larger than the turbulent time scales in order for the averaging procedure in Eq. (17) to be justified. In the initial period comparable to the averaging time scale $T$ the averaged LES quantities are not reliable due to the limited time series available for averaging. Therefore, the coupling is disabled for the sake of stability and the LES/RANS cell classification is not performed (and not necessary either). With the overall limitation of the current hybrid solver in mind, this practice of starting hybrid simulations is acceptable. After this initial transient period is passed, the LES/RANS cell detection can be safely performed based on the averaged LES quantities, which should be rather reliable.

\section{Conclusions}

In this work, we investigated a resolution evaluation criterion based on the ratio between turbulent length-scales and grid spacing, both of which are cell-based quantities, making it very convenient for dynamic resolution evaluation in hybrid LES/RANS simulations. The length-scale based criterion found in the literature is modified to distinguish the resolution in different directions and to have similar physical interpretation as the criterion based on two-point correlations. This modified length-scale criterion and the two-point correlation criterion are both assessed for plane channel flow of $R e_{\tau}=4000$ with meshes of six different resolutions. Analysis suggests that the two resolution indicators give qualitatively similar predictions of the mesh resolution, and they both have physical interpretations consistent to the existing knowledge on turbulent channel flows. Simulations of flow over periodic hills confirmed that the modified length-scale resolution indicator gives correct responses over mesh refinements. It is further demonstrated that compared to criteria based on the percentage of resolved turbulent quantities, the modified length-scale criterion is less susceptible to the variation of model constants, which we argue is a desirable property for resolution criteria.

The length-scale based resolution criterion was implemented into a consistent dual-mesh hybrid LES/RANS framework and used to simulate flows over periodic hills. The results demonstrated that the interactions between resolution evaluations and the solutions are moderate, which is another desirable feature for resolution indicators. The comparison with benchmark results showed that the hybrid solver using the dynamic resolution criterion lead to improved results compared with pure under-resolved LES on the same mesh.

This study aims to investigate the possibility of determining local resolution adequacy based on cell values of turbulent quantities. Although the main purpose of this resolution evaluation is the classification of well-resolved (LES) regions and under-resolved (RANS) 
regions in hybrid LES/RANS methods, in principle this methodology of resolution evaluation can also be used in pure LES simulations as guidance for adaptive grid refinement; i.e., the mesh could be refined or coarsened during the simulations.

However, there are many open issues associated with this length-scale based resolution indicator, including the choice of a functional form of the vector compared to a threshold value, the choice of threshold values for determining resolution adequacy, the effects of frame-dependence, and the resolution evaluation in regions with strong instability and recirculation. Unfortunately, these difficulties are common to most other resolution indicators as well. Therefore, reliable and dynamic evaluation of resolution adequacy of individual cells in hybrid LES/RANS simulations remains a challenging task, and further research is warranted. In addition, this work is mainly concerned with developing and evaluating a reliable resolution criterion for hybrid LES/RANS simulations, which are mostly used to overcome the restrictive resolution requirement for LES when used in wall-bounded flows. Therefore, we focused on the canonical test cases of wall-bounded flows; the test cases of shear flows are not investigated in the current study and are left for future research.

Acknowledgment HX would like to acknowledge the financial support from the Commission for Technology and Innovation (CTI) of Switzerland. The computational resources used for this project were provided by the Brutus Cluster at ETH Zürich, and by the Advanced Research Computing (ARC) of Virginia Tech, which are gratefully acknowledged. We thank Prof. M. Breuer at the Helmut-Schmidt Universitaet Hamburg for providing us the benchmark data for comparison.

\section{References}

1. Chapman, D.R.: Computational aerodynamics: Development and outlook. AIAA J. 17, 1293-313 (1979)

2. Xiao, H., Jenny, P.: A consistent dual-mesh framework for hybrid LES/RANSmodeling. J. Comput. Phys. 231(4), 1848-1865 (2012)

3. Gant, S.E.: Reliability issues of LES-related approaches in an industrial context. Flow Turbul. Combust. 84, 325-335 (2010)

4. Celik, I.B., Cehreli, Z.N., Yavuz, I.: Index of resolution quality for large eddy simulations. J. Fluids Eng., 949-958 (2005)

5. Klein, M.: An attempt to assess the quality of large eddy simulations in the context of implicit filtering. Flow Turbul. Comathbfust. 75, 131-147 (2005)

6. Grötzbach, G.: Revisiting the resolution requirements for turbulence simulations in nuclear heat transfer. Nucl. Eng. Des. 241(11), 4347-4632 (2011)

7. Sagaut, P.: Large Eddy Simulations for Incompressible Flows: An Introduction. Springer (2006)

8. Geurts, B.J., Fröhlich, J.: A framework for predicting accuracy limitations in large eddy simulation. Phys. Fluids 14, 41-44 (2002)

9. Davidson, L.: Large eddy simulations: How to evaluate resolution. Int. J. Heat Fluid Flow 30, 1016-1025 (2009)

10. Davidson, L.: How to estimate the resolution of an LES of recirculating flow. In: Quality and Reliability of Large-Eddy Simulations II, pp. 269-286. Springer (2010)

11. Pope, S.B.: Turbulent Flows. Cambridge University Press, Cambridge (2000)

12. Breuer, M., Jaffrezic, B., Arora, K.: Hybrid LES-RANS technique based on a one-equation near-wall model. Theor. Comput. Fluid Dynamics 22, 157-187 (2008)

13. OpenCFD Ltd.: The open source CFD toolbox. www.openfoam.com (2011)

14. Xiao, H., Wild, M., Jenny, P.: Preliminary evaluation and applications of a consistent hybrid LES-RANS method. In: Fu, S., et al. (eds.) Progress in Hybrid RANSLES Modeling, NNFM (117), pp. 91-100. Springer (2011)

15. Issa, R.I.: Solution of the implicitly discretised fluid flow equations by operator-splitting. J. Comput. Phys. 62, 40-65 (1986)

16. Rhie, C.M., Chow, W.L.: A numerical study of the turbulent flow past an isolated airfoil with trailing edge separation. AIAA 21(11), 1525-1532 (1983) 
17. Yoshizawa, A., Horiuti, K.: A statistically-derived subgrid-scale kinetic energy model for the large-eddysimulation of turbulent flows. J. Phys. Soc. Jpn. 54, 2834-2839 (1985)

18. Jakirlić, S., Kniesner, B., Kadavelil, G., Gnirß, M.: Experimental and computational investigations of flow and mixing in a single-annular comathbfustor configuration. Flow Turbul. Comathbfust. 83, 425448 (2009)

19. Moin, P., Kim, J.: Numerical investigation of turbulent channel flow. J. Fluid Mech. 118, 341-377 (1982)

20. Saric, S., Jakirlic, S., Breuer, M., Jaffrezic, B., Deng, G., Chikhaoui, O., Fröhlich, J., von Terzi, D., Manhart, M., Peller, N.: Evaluation of detached eddy simulations for predicting the flow over periodic hills. In: ESAIM: Proceedings, vol. 16, pp. 133-145. doi:10.1051/proc:2007016 (2007)

21. Temmerman, L., Leschziner, M.A.: Flow over 2D periodic hills. http://cfd.mace.manchester.ac.uk/twiki/ bin/view/CfdTm/TestCase014

22. Addad, Y., Laurence, D., Talotte, C., Jacob, M.C.: Large eddy simulation of a forward-backward facing step for acoustic source identification. Int. J. Heat Fluid Flow 24(4), 562-571 (2003)

23. Breuer, M., Peller, N., Rapp, C., Manhart, M.: Flow over periodic hills: Numerical and experimental study in a wide range of Reynolds numbers. Comput. Fluids 38(2), 433-457 (2009)

24. Durbin, P.A.: A Reynolds stress model for near-wall turbulence. J. Fluid Mech. 249, 465-498 (1993)

25. Nicoud, F., Ducros, F.: Subgrid-scale stress modelling based on the square of the velocity gradient tensor. Flow Turbul. Comathbfust. 62, 183-200 (1999)

26. Kim, J., Moin, P., Moser, R.: Turbulence statistics in fully developed channel flow at low Reynolds number. J Fluid Mech. 177, 133-166 (1987) 